\title{
Eating disorders in the Arab world: a literature review
}

\author{
Bernou Melisse ${ }^{1,2,3^{*}}$ (D), Edwin de Beurs ${ }^{4,5}$ and Eric F. van Furth 2,3
}

\begin{abstract}
Background: The prevalence of eating disorders has been assumed to be low in the Arab world, due to the alleged absence of the thin ideal. However, the Arab world is undergoing rapid sociocultural changes, and there are reports of an increase of the desire to be thin. This literature review therefore provides point-prevalence of Arabs at high risk for eating disorders, and a comprehensive synthesis of correlates of eating disorder symptoms, eating disorder-related variables and of a high risk for eating disorders.

Method: Several electronic databases were searched for published, peer-reviewed studies between 1986 and 2019 involving several key terms. From 317 screened studies, 81, mainly cross-sectional, were included. Preferred Reporting Items for Systematic reviews and meta-analyses was used as guidance and the quality of studies were assessed using the Newcastle-Ottawa scale.
\end{abstract}

Results: Estimates of individuals at high risk for eating disorders ranged from 2 to $54.8 \%$. The eating disorderrelated variables identified were desire to be thin, body dissatisfaction, disturbed-, and dieting- eating behavior. Identified correlates were increased affluence, media use, western influences, and obesity. An additional finding was that in some cases eating disorders were expressed somatically rather than psychiatrically.

Discussion: In the Arab world, females were most at risk for eating disorders and eating disorder symptoms. Sociocultural changes gave rise to the thin ideal and the prevalence of obesity, increasing the risk for the development of eating disorder-related variables and eating disorders. The literature on eating disorders in the Arab world suffers from potential limitations due to the use of non-validated assessment tools. Further research is necessary, particularly on the development and validation of a culturally sensitive assessment tool. Improved knowledge is likely to increase the number of people seeking treatment and decrease the stigma of psychotherapy.

Keywords: Arab, Eating disorders, Prevalence, Eating disorder-related variables, Desire to be thin, Correlates

\section{Plain English summary}

In the Arab world a curvy body was perceived as fertile, so eating disorders were assumed to be rare. In recent years, however, due to rapid sociocultural changes, Western influences, media use, and increased affluence, Arabs have started to admire a thinner body. Consequently, research

\footnotetext{
* Correspondence: bernoumelisse@outlook.com

'Novarum Center for Eating Disorders \& Obesity, Jacob Obrechtstraat 92, 1071, KR, Amsterdam, the Netherlands

${ }^{2}$ Rivierduinen Eating Disorders Ursula, Sandifortdreef 19, 2333 ZZ Leiden, Netherlands

Full list of author information is available at the end of the article
}

has now begun to address the existence of eating disorders in the Arab world. The increased popularity of the desire to be thin has been associated with soft symptoms such as disturbed eating behavior, body dissatisfaction and dieting behavior. In addition to Arabs at risk for eating disorders, eating disorder symptoms identified include binge eating, self- induced vomiting, and laxative use. Sometimes, eating disorders manifest themselves in a different way in the Arab world: some Arabs have expressed eating disorders somatically, as nausea, stomach ache, and so on, rather than psychiatrically. There is a need for assessment tools to be adjusted to the Arab culture. Improved knowledge

(c) The Author(s). 2020 Open Access This article is licensed under a Creative Commons Attribution 4.0 International License, which permits use, sharing, adaptation, distribution and reproduction in any medium or format, as long as you give appropriate credit to the original author(s) and the source, provide a link to the Creative Commons licence, and indicate if changes were made. The images or other third party material in this article are included in the article's Creative Commons licence, unless indicated otherwise in a credit line to the material. If material is not included in the article's Creative Commons licence and your intended use is not permitted by statutory regulation or exceeds the permitted use, you will need to obtain permission directly from the copyright holder. To view a copy of this licence, visit http://creativecommons.org/licenses/by/4.0/ The Creative Commons Public Domain Dedication waiver (http://creativecommons.org/publicdomain/zero/1.0/) applies to the data made available in this article, unless otherwise stated in a credit line to the data. 
will facilitate recognition of eating disorders, encourage people to seek treatment and decrease the stigma of psychotherapy.

\section{Background}

Eating disorders (EDs) have a significant impact on the well-being of affected individuals [1]. This includes comorbid obesity [2], depressive symptoms, anxiety [3] substance abuse, suicide attempts [4], and high rates of mortality and relapse [5]. Since EDs have historically been associated with Caucasian females in developed Western countries with high socio-economic status [6], they have been perceived as culturally bound syndromes [7]. Taking this in consideration most studies regarding EDs have been heavily concentrated in Western countries [8].

Cultural factors are essential to understanding the development of EDs [9] and the main feature identified associated with EDs in the West is a thin body ideal [6], which is presented to the society as achievable by dieting and exercising [8]. Traditional Arab notions of beauty are different, with the curvy body ideal associated with fertility and wealth [10] EDs were assumed not to afflict Arabs $^{1}[10,11]$ hence EDs were not reported in the Arab world until 1986 [12]. However, from then on [10, 11] reports on the thin ideal in the Arab world steadily increased [13-15].

Recent studies have shown that EDs occur globally [16, 17], EDs occur particularly in cultures in transition as they adopt Western values $[7,18]$, illuminating the interplay between culture and psychopathology [19]. This is relevant to the Arab world, since the oil boom of the 1970s and the consequent increased affluence [20,21], it has been undergoing rapid sociocultural and socioeconomic changes [14, 22 . The oil boom led to the arrival of Western companies, Western expatriates $[18,23]$, and to increased exposure to Western culture [8].

The sociocultural changes associated with acculturation include adopting the language, lifestyle, values, and beliefs of other cultures [24]. In the Arab world, increased exposure to Western media [20, 25-27], and increased contact with expatriates [28, 29] has also led to a rise in the popularity of the thin ideal $[10,30]$, and to increased levels of dieting, body dissatisfaction and EDs [8]. However, the theory of Westernization assumes that the Western culture is transferrable to Arab cultures [31], the oil boom has also been associated with elevated levels of industrialization [18] including increased technology, affluence, and higher education [32, 33]. Industrialization in particular has coincided with changes in the types of food available [7, 34], and these are instrumental in a rise in obesity $[28,35,36]$. Together, these changes have contributed to elevated levels of non-communicable diseases such as diabetes mellitus, hypertension [20], and psychological problems [37] including ED-related variables and EDs [18, 38-40].

Kraemer and colleagues [41-43] have provided us with a terminology to address how culture and EDs may be associated. An important distinction is that between a correlate and a risk factor. A correlate is a potential risk factor that is measured at the same time as an ED. The correlate and ED are associated with each other, but precedence of the correlate to the onset of an ED is not proven [41, 42]. Therefore correlates cannot be defined as risk factors. Correlates are identified in cross sectional studies [41-43]. Currently identified correlates are increased affluence [6, 43], obesity [44, 45], and societal changes such as industrialization [46], globalization [6], and acculturation $[38,46]$. If it is established that an associated variable precedes the onset of an ED, this variable may be indicated as a risk factor [42]: gender is an example [41]. A risk factor identifies individuals who are at elevated risk for future emergence of an ED [43], and risk factors can best be identified by longitudinal studies $[41,42]$. Initiation of the onset of an ED can be indicated by ED-related variables [43]. ED-related variables are an early sign of an ED which indicate vulnerability to develop an ED. However, on their own ED-related variables do not meet the criteria for an ED as described in the DSM-5 [47]. Examples of ED-related variables are disturbed eating behavior, self-reported dieting, an elevated desire to be thin, and body dissatisfaction [41].

To distinguish between various levels of ED-pathology, commonly the following types are described: ED symptoms, at high risk for ED and full syndrome ED [41-43]. ED symptoms are symptoms that are defining features of an ED [43] such as binge eating, and compensatory behaviors, which can be measured by self-report and clinical interviews. If the frequency of ED symptoms meets the criteria of an ED, including clinically significant functional impairment [6], an individual can be classified as suffering from a full syndrome ED [43, 47]. Full syndrome EDs are best diagnosed with a clinical interview [43]. Individuals who score above a clinical cut-off on an ED-screening instrument are considered to be at high risk for an ED $[43,48]$.

This review will differentiate between ED-related variables, ED symptoms, and high risk for EDs. Arabs with elevated levels of ED-related variables and ED symptoms are at risk for EDs, as are Arabs who score above a clinical cut-off on an ED-screening instrument, who will be referred to as Arabs at high risk for EDs. Correlates of ED-related variables, ED symptoms, and scores above a clinical cut-off on an ED-screening instrument will also be identified. Identification of correlates and ED-related variables is likely to facilitate identification of risk groups for prevention programs $[43,49]$. At the same time, preventative and treatment programs cannot be copied 
from Western societies [50]. They need to be adapted to the relevant sociocultural context $[51,52]$.

Although EDs have been reported in the Arab world $[28,29,36,53-55]$, there is limited valid data [56], and the substantial differences in the expression of EDs among different cultures $[7,52,57,58]$ are yet to be taken into account. For example, somatic expression of EDs is more likely in the Arab world than in the West [52, 58]. Some Arabs attribute a restrictive food pattern to somatic complaints [59], while there are also reports of body dissatisfaction and fear of fatness [52, 58]. Examples of somatic complaints are stomach ache, feeling bloated or an absence of appetite [59]. In addition, perceptions of body image also differ between cultures [52].

There are a number of comprehensive reviews of the nature of EDs in Western countries [5, 43, 51, 60, 61]. However, as the Arab world has undergone rapid sociocultural changes [14, 22], EDs could be more culturally reactive than culturally bound [8]. An overview of EDs in the Arab world is needed. Unfortunately, due to a lack of appropriate and valid data, it is not feasible to examine how the prevalence of EDs in the Arab world has changed over the years. Still, data from ED-screening instruments can help identify individuals at high risk for EDs, and enable examination of associations between ED-related variables, correlates and ED symptoms. In this review, we therefore aim to provide estimates regarding the point-prevalence of Arabs at high risk for EDs, with ED symptoms above threshold values for caseness, and to provide a comprehensive synthesis of relevant studies of correlates and ED-related variables. The countries included here are those together referred to as "the Arab world", which is a part of the Eastern Mediterranean Region [62], also referred to as the Middle East [63]. Non-Arab countries such as Cyprus and Turkey are excluded [64]. To the best of our knowledge, this is the first review summarizing EDs in the Arab world.

\section{Method}

\section{Search strategy}

The primary search was conducted in Web of Science, PubMed (Medline) and Google Scholar databases from 1986 up to July 2019 by BM, and involved key terms related to ED prevalence in the Arab world. All combinations of ("Eating disorders" OR "Anorexia Nervosa" OR "Bulimia Nervosa" OR "Binge Eating Disorder" OR "Disturbed Eating Behavior" OR "Eating Attitudes" OR "Dieting" OR "Body Image" OR" Body Satisfaction" OR "Obesity") AND ("Arab" OR "Middle East" OR "Gulf" OR "United Arab Emirates (UAE)" OR "Saudi" OR "Oman" OR "Qatar" OR "Bahrain" OR "Kuwait" OR "Lebanon" OR "Palestine" OR "Jordan") were searched. To be included, articles had to be peer reviewed. This resulted in 14,656 hits. Subsequently, more records were added from other sources $(n=13)$. After duplicates were removed, 14,629 studies remained. The titles were then screened for eligibility. Eligible studies were conducted in Arab countries in the Middle East, providing estimates regarding the point prevalence of individuals at high risk for EDs, ED symptoms and ED-related variables, and reporting correlates of scores above a clinical cut-off in ED-screening instruments, ED symptoms, and ED-related variables. Records $(n=14,312)$ studying, for example, diabetes mellitus, metabolic syndrome or nutrition related to other diseases, were excluded. When there was doubt, the abstract and discussion section were further screened. This resulted in 72 relevant studies, plus 9 additional studies identified from their lists of reference. The additional sources of studies were four websites involving statistical data such as GCC stat (Fig. 1). Most studies were conducted in the UAE $(n=$ $11)$, followed by Jordan and Saudi Arabia $(n=7)$. A further six studies were conducted in Kuwait, five in Egypt, four each in Oman, and Qatar. Three among Palestinian populations, two each in Bahrain, Lebanon, Iran, and one each in Algeria, and Libya.

\section{Inclusion criteria}

Inclusion criteria were that the studies involved Arab Middle Eastern countries [63] of the Eastern Mediterranean Region [62]. These are West Asian countries (Lebanon, Jordan, Iran), the Gulf (Bahrain, Kuwait, Qatar, UAE, Saudi Arabia, Oman), Egypt, and Palestinian populations. Though, a non-Arab country, Iran is included because of the several cultural similarities (e.g. hijab and full body coverage for females in public) [64]. All studies investigated at least one ED symptom or ED-related variable. In cases where obesity was studied, study of at least one additional ED behavior was required. There were no restrictions on age or gender. All studies reported point prevalence, the prevalence at a certain point in time [51]. Additional studies examined correlates of EDs, ED symptoms or ED-related variables.

\section{Exclusion criteria}

Studies involving non-Middle Eastern countries, and non-Arab Middle Eastern countries were excluded [63], as were studies focusing only on obesity, and qualitative studies without empirical data.

\section{Assessments}

While correlates and ED-related variables can be accurately measured by self-report [43], diagnostic interviews are preferable for ED symptoms. Since diagnostic concordance between diagnostic interviews and self-report is moderate at best [65], and EDs can best be assessed by diagnostic interviews [43], questionnaires including the SCOFF, EAT, and EDE-Q may at best provide 


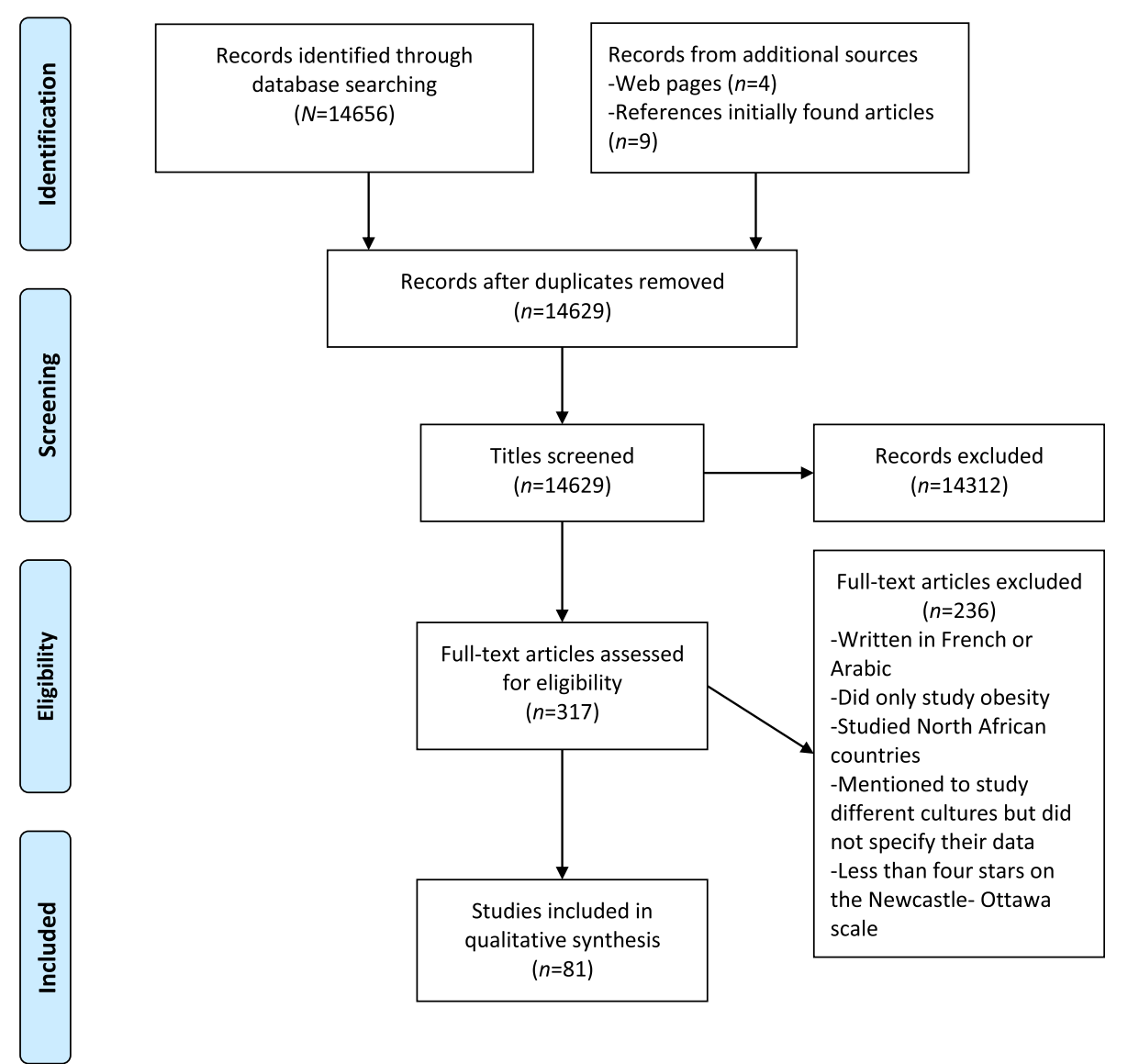

Fig. 1 Flow chart of study selection process based on PRISMA

prevalence estimates of Arabs at high risk for EDs, indicated by elevated scores at ED-screening instruments. However, since for several Arab populations norms of ED-screening instruments were not available, norms of either Western populations or other Arab populations were used. Therefore, these instruments indicated elevated scores on ED-screening instruments in the target country using the norms of another country. In this study, Arabs who scored above a clinical cut-off are referred to as Arabs at high risk for EDs, regardless of the norms used.

\section{Data abstraction and quality assessment}

This review is based on studies published in English between 1986 and 2019; a large timeframe was chosen due to the limited number of studies on EDs conducted in the Arab world. Since only studies on point prevalence were found and none on incidence rate and lifetime prevalence, prevalence refers to point prevalence. Most of the studies were published in peer reviewed journals. Two theses were also included $[66,67]$. In total, 81 studies met the inclusion criteria. Almost all the studies were quantitative; most were cross sectional, although three were case control studies $[35,68,69]$. One validation study was included
[54], and longitudinal data were not available. Eight cross sectional studies involved cross cultural aspects [11, 23, $26,39,52,58,64,70]$. Author BM assessed the risk of bias on study validity. Discrepancies or uncertainties were resolved by discussion with either the second or the third author. Data extraction was done independently by BM, taking into consideration study characteristics, demographics, study results, and key study outcomes. Quality of included studies was assessed using the NewcastleOttawa scale [71], a seven item scale that investigates power, research design, sample, recruitment, and statistical analysis (see supplementary material). The maximum score was 10 stars, based on selection (maximum five stars), comparability (maximum two stars) and outcome (maximum three stars). Only studies with at least four stars were included in the review. Preferred Reporting Items for Systematic reviews and Meta-Analyses (PRIS MA) was used as guidance (Fig. 1) [72].

\section{Results}

Quality assessment

Of 317 studies assessed for eligibility, 81 studies met the minimum criteria of four stars on the Newcastle Ottawa 
scale (Fig. 1) [71]. However, the 81 studies included had several limitations, these studies either involved a selected group of users or had a selective sample. For example, the association between media and EDs in Kuwait was assessed only on female adolescents at one university [73], disturbed eating behavior only amongst adolescent girls in the UAE, and only among the inhabitants of al Ain province [20], and in Saudi Arabia, only in the city of Hail [67], all involving academically inclined schoolgirls [39]. Most of the studies involved a population of university students [74]. In addition, reporting in some studies was incomplete (lack of demographic data, such as mean age, and marital status) [67, 75]. In other studies mean and SD [26, 29, 39, 54, 55, 64, 76-79] or percentage that scored above the clinical cutoff $[52,58,64]$ on the ED-screening instrument were not reported.

Another limitation was that power calculations were not reported in some studies, so their sample size was not justified and was low. This was the case $(N=100)$ in an Omani [29], a Saudi [67], and $(N=104)$ an Iranian [64] sample. Studies examining the association between Westernization and ED had only small samples $(N<100)$ in the UAE [40], and in Iran [64].Another limitation was that several studies used non-validated ED-screening instruments. Several studies, for instance, translated the EAT-40 without adaptations, and used Western norms $[20,28,39]$. In another study on ED in Saudi Arabia, the EAT-26 was translated without adaptations, and applied using Western norms [67]. The Saudi version of the EAT-26, which included the Western norms was also used in non-Saudi countries [14, 18, 26, 40, 52, 53, 58, $74,79,80]$. Other studies investigated adults using a version of the Saudi EAT-26 validated in a high school population [26]. Musaiger and Al-Mannai [73] also examined a Kuwaiti sample using questions validated in a Bahraini sample. In addition, two studies assessed disturbed eating behavior among adolescent Omani and European expatriates residing in Oman using the EAT26 and the EDI-2. These studies found that Omanis had significant higher EAT26 scores, while European expatriates had significantly higher EDI-2 scores. Omanis and European expatriates scored significantly different on both ED-screening instruments [52, 58], possibly due to the fact that a Saudi version including Saudi norms were used for the EAT26 and EDI-2 [78, 81]. Several studies also failed to provide essential information on the norms used, and some studies provided no information at all. One study, in Kuwait [75], did not provide information about how their statistical analysis was conducted. Furthermore, none of the studies on binge eating behavior mentioned the frequency of binge eating episodes, or whether the binges were of a subjective or objective nature $[29,70,74,77]$. All the studies discussed relied on self-report measures, which are prone to socially desirable responding [75]. The collectivistic culture of the Arab world makes people hesitant to report individual desires [76], so the use of self-report measures makes studies vulnerable to under- or overestimation of complaints [77].

In conclusion, most of the studies included in this synthesis have limitations. These include the absence of psychometric validation of assessment tools for the Arab world, and the absence of necessary data such as demographic data, power calculation proper assessment, and description of ED-pathology.

\section{Eating disorder-related variables}

Table 1 presents a summary of 19 studies including EDrelated variables. These were: desire to be thin, body dissatisfaction, disturbed eating behavior, and dieting [43]. Disturbed eating behavior was involved in nine studies, body dissatisfaction in ten studies, desire to be thin in seven, and dieting in five cross sectional studies. EDrelated variables were identified by self-report in all studies, except for one, which used both interview ánd self- report data [39]. All ED-related variables were mainly assessed in female adolescents.

\section{Disturbed eating behavior}

Overall, the evidence suggested that disturbed eating behavior increases with age during adolescence and decreases during adulthood [20, 26, 29, 52, 53, 58, 67]. Females [26] and Saudis [67] appeared to be most at risk of disturbed eating behavior [14, 26, 90].

\section{Dieting}

The findings suggested that, as a whole, approximately $40 \%$ of the Arab population is on a diet [20,35, 70, 88], and this is true for adults and adolescents of both genders $[20,35,70]$. Saudis were at the lowest risk of dieting [13].

\section{Body dissatisfaction}

Females were more vulnerable to body dissatisfaction than male Arabs [10, 82]. Higher BMI was associated with greater body dissatisfaction $[64,75,83-85,91]$.

\section{Desire to be thin}

Though findings were quite inconsistent, overall Arabs from the UAE had the highest desire to be thin [13-15], whereas Qataris appeared to be minimally affected by the thin ideal [15]. A risk factor of the desire to be thin was adulthood over adolescence [10, 13-15, 20, 87, 89].

\section{Eating disorder symptoms}

Table 2 provides a summary of 11 studies that examined eating disorder symptoms in the Arab world. Binge eating and restrained eating behavior were each identified 
Table 1 Summary of studies reporting eating disorder-related variables in the Arab world

\begin{tabular}{|c|c|c|c|c|c|c|}
\hline $\begin{array}{l}\text { Country/ } \\
\text { Population }\end{array}$ & $\begin{array}{l}\text { Authors } \\
\text { (year) }\end{array}$ & Participants & $\begin{array}{l}\text { Study } \\
\text { design }\end{array}$ & Measures & $\begin{array}{l}\text { Eating disorder-related } \\
\text { variables }\end{array}$ & $M(S D) / O R[95 \% \mathrm{Cl}] / r$ \\
\hline $\begin{array}{l}\text { Algeria, Jordan, } \\
\text { Kuwait, Libya, } \\
\text { Palestinians } \\
\text { residing in al- } \\
\text { Khalil, Syria, } \\
\text { UAE }\end{array}$ & $\begin{array}{l}\text { Musaiger } \\
\text { et al., (2013) } \\
\text { [26] }\end{array}$ & $\begin{array}{l}N=4698,2240 \\
\text { male, } 2458 \\
\text { female, age } 15- \\
18\end{array}$ & $\begin{array}{l}\text { Cross } \\
\text { sectional }\end{array}$ & EAT 26 & $\begin{array}{l}\text { Disturbed eating behavior: } \\
\text { twice as high in females than } \\
\text { in males in Jordan, Libya, } \\
\text { Palestinians residing in al-Khalil } \\
\text { and Syria }\end{array}$ & $\begin{array}{l}\text { Disturbed eating behavior: } \\
(p<0.000), \text { males as reference } \\
\text { Jordan OR } 2.96[2.19-4.01] \\
\text { Libya OR 2.02 [1.37-2.98] } \\
\text { Palestinians residing in al-Khalil } \\
\text { OR 2.11 [1.39-3.22] } \\
\text { Syria OR 2.75 [2.02-3.77] }\end{array}$ \\
\hline Bahrain & $\begin{array}{l}\text { Al-Sendi, } \\
\text { Shetty, \& } \\
\text { Musaiger } \\
\text { (2004) [82] }\end{array}$ & $\begin{array}{l}N=504,249 \\
\text { male, } 257 \text { female, } \\
\text { age } 12-17\end{array}$ & $\begin{array}{l}\text { Cross } \\
\text { sectional }\end{array}$ & $\begin{array}{l}\text { FRS (self- } \\
\text { developed) }\end{array}$ & $\begin{array}{l}\text { Body dissatisfaction: female: } \\
50 \% \text {, male: } 30 \%\end{array}$ & not reported \\
\hline Egypt & $\begin{array}{l}\text { Ford, Dolan, \& } \\
\text { Evans (1990) } \\
\text { [10] }\end{array}$ & $\begin{array}{l}N=218,61 \text { male, } \\
\text { mean age = 20.0, } \\
169 \text { female, mean } \\
\text { age }=19.5, \\
\text { university } \\
\text { students }\end{array}$ & $\begin{array}{l}\text { Cross } \\
\text { sectional }\end{array}$ & $\begin{array}{l}\text { FRS (self- } \\
\text { developed) }\end{array}$ & $\begin{array}{l}\text { Thin ideal: female: ideal shape } \\
\text { significantly thinner than their } \\
\text { actual shape; male \& female: } \\
\text { preference for thinness }\end{array}$ & $\begin{array}{l}\text { Discrepancy between current } \\
\text { and ideal figure: } t=3.67, p= \\
0.001 \text {. Mean discrepancy: } \\
\text { female } 0.56-1.02 \text {, male }-0.02- \\
1.00 \\
M(S D)=\text { Female current } 3.75 \\
(0.9) \text {, ideal } 3.19(0.9) \text {. Male } \\
\text { current } 4.18(1.1) \text {, ideal } 4.2(0.7)\end{array}$ \\
\hline Jordan & $\begin{array}{l}\text { Madanat, } \\
\text { Hawks, \& } \\
\text { Angeles } \\
\text { (2011) [83] }\end{array}$ & $N=800$, female & $\begin{array}{l}\text { Cross } \\
\text { sectional }\end{array}$ & $\begin{array}{l}\text { 9- figure } \\
\text { silhouettes }\end{array}$ & Body dissatisfaction: 66\% & not reported \\
\hline Jordan & $\begin{array}{l}\text { Mousa, } \\
\text { Mashal, Al- } \\
\text { Domi, \& Jibril } \\
\text { (2010) [84] }\end{array}$ & $\begin{array}{l}N=326, \text { female, } \\
\text { age: } 10-16\end{array}$ & $\begin{array}{l}\text { Cross } \\
\text { sectional }\end{array}$ & $\begin{array}{l}\text { EAT } 26 \\
\text { BSQ |Western } \\
\text { norms }\end{array}$ & $\begin{array}{l}\text { Body dissatisfaction: } 21.2 \% \text {. } \\
\text { Association between EAT and } \\
\text { BSQ. }\end{array}$ & $\begin{array}{l}\text { BSQ: } M(S D)=79.1(34.5), 21.2 \% \\
\text { above cutoff } \\
X 2(1,326)=104.8, p<0.01\end{array}$ \\
\hline Jordan & $\begin{array}{l}\text { Zawawi } \\
\text { (2014) [85] }\end{array}$ & $\begin{array}{l}N=170, \text { female, } \\
\text { age: } 20-55 \\
\text { fitness center } \\
\text { users }\end{array}$ & $\begin{array}{l}\text { Cross } \\
\text { sectional }\end{array}$ & BSQ & Body dissatisfaction: 31.01\% & $M(S D)=3.19(10.3)$ \\
\hline Kuwait & $\begin{array}{l}\text { Ebrahim, } \\
\text { Alkazemi, } \\
\text { Zafar, \& } \\
\text { Kubow (2019) } \\
\text { [86] }\end{array}$ & $\begin{array}{l}N=400, \text { Male, } \\
\text { university } \\
\text { students }\end{array}$ & $\begin{array}{l}\text { Cross } \\
\text { sectional }\end{array}$ & $\begin{array}{l}\text { Body Builder } \\
\text { Image Grid }\end{array}$ & $\begin{array}{l}\text { Body dissatisfaction: } 69 \% \text {, desire } \\
\text { to lose body fat associated } \\
\text { with disordered eating } \\
\text { attitudes }\end{array}$ & $\begin{array}{l}\mathrm{OR}=1.898[1.214-2.967], p= \\
0.005\end{array}$ \\
\hline Kuwait & $\begin{array}{l}\text { Musaiger \& } \\
\text { Al- Mannai } \\
\text { (2013) [26] }\end{array}$ & $\begin{array}{l}N=228, \text { female, } \\
\text { university } \\
\text { students, age } 19- \\
25\end{array}$ & $\begin{array}{l}\text { Cross } \\
\text { sectional }\end{array}$ & $\begin{array}{l}\text { Questions } \\
\text { validated by Field } \\
\text { et al., 2005, } \\
\text { translated into } \\
\text { Arabic }\end{array}$ & $\begin{array}{l}\text { Body dissatisfaction: non-obese: } \\
30 \% \text {, obese: } 81 \% .21 .6 \% \text { of non- } \\
\text { obese perceived themselves as } \\
\text { overweight }\end{array}$ & not reported \\
\hline Lebanon & $\begin{array}{l}\text { Zeeni, } \\
\text { Gharibeh, \& } \\
\text { Katsounari } \\
\text { (2013) [23] }\end{array}$ & $\begin{array}{l}N=400, \text { female, } \\
\text { university } \\
\text { students in } \\
\text { Cyprus }(n=200) \\
\text { and Lebanon } \\
(n=200)\end{array}$ & $\begin{array}{l}\text { Cross } \\
\text { sectional }\end{array}$ & $\begin{array}{l}\text { Dutch eating } \\
\text { behavior } \\
\text { questionnaire }\end{array}$ & $\begin{array}{l}\text { Association between restrained } \\
\text { and emotional eating }\end{array}$ & $\begin{array}{l}M(S D) \text { : restrained }=29.20 \\
(0.71), \text { emotional eating }=37.76 \\
(0.98) \text {, external eating }=33.33 \\
(0.51), p<0.05\end{array}$ \\
\hline Qatar & $\begin{array}{l}\text { Bener, Kamal, } \\
\text { Tewfik, \& } \\
\text { Sabuncuoglu } \\
\text { (2006) [35] }\end{array}$ & $\begin{array}{l}N=800, \text { male } \\
\text { age } 14-19\end{array}$ & $\begin{array}{l}\text { Case } \\
\text { control } \\
\text { (dieting) }\end{array}$ & $\begin{array}{l}\text { Adolescent dieting } \\
\text { scale } \\
\text { Self- reporting } \\
\text { questionnaire }\end{array}$ & Extreme dieting: $10.1 \%$ & not reported \\
\hline Qatar & $\begin{array}{l}\text { Musaiger, } \\
\text { Shahbeek, \& } \\
\text { Al-Mannai } \\
\text { (2004) [15] }\end{array}$ & $\begin{array}{l}N=535, \text { male, } \\
\text { age } 20-67, \\
\text { primary health } \\
\text { care center } \\
\text { visitors }\end{array}$ & $\begin{array}{l}\text { Cross } \\
\text { sectional }\end{array}$ & $\begin{array}{l}\text { 9- figure } \\
\text { silhouettes }\end{array}$ & $\begin{array}{l}\text { Desire to be thin: } 21.6 \% \text {, low } \\
\text { education } 40 \% \text {, mid-level edu- } \\
\text { cation } 45 \% \text {, high education } \\
53 \% \text {. Desire to be thin was as- } \\
\text { sociated with age and } \\
\text { education }\end{array}$ & $\begin{array}{l}\text { Thin ideal, education: } p= \\
0.0001 \text {, age }>40 \text { years } p= \\
0.0001\end{array}$ \\
\hline Saudi Arabia & $\begin{array}{l}\text { Al- Subaie } \\
\text { (2000) [87] }\end{array}$ & $\begin{array}{l}N=1179, \text { female, } \\
\text { mean age }=16.1\end{array}$ & $\begin{array}{l}\text { Cross } \\
\text { sectional }\end{array}$ & EDI 2 DT & Desire to be thin: $15.9 \%$ & $M=6.7, S D$ not reported \\
\hline Saudi Arabia & $\begin{array}{l}\text { Fallatah, Al- } \\
\text { Hemairy, \& Al- }\end{array}$ & $\begin{array}{l}N=425, \text { female, } \\
\text { age } 15-18\end{array}$ & $\begin{array}{l}\text { Cross } \\
\text { sectional }\end{array}$ & EAT 26 & $\begin{array}{l}\text { Prevalence of dieting not } \\
\text { reported }\end{array}$ & Dieting: 9.38 (7.0) \\
\hline
\end{tabular}


Table 1 Summary of studies reporting eating disorder-related variables in the Arab world (Continued)

\begin{tabular}{|c|c|c|c|c|c|c|}
\hline $\begin{array}{l}\text { Country/ } \\
\text { Population }\end{array}$ & $\begin{array}{l}\text { Authors } \\
\text { (year) }\end{array}$ & Participants & $\begin{array}{l}\text { Study } \\
\text { design }\end{array}$ & Measures & $\begin{array}{l}\text { Eating disorder-related } \\
\text { variables }\end{array}$ & $M(S D) / O R[95 \% \mathrm{Cl}] / r$ \\
\hline & $\begin{array}{l}\text { Ghamidi } \\
\text { (2015) [66] }\end{array}$ & & & & & \\
\hline UAE & $\begin{array}{l}\text { Eapen, } \\
\text { Mabrouk, \& } \\
\text { bin Othman } \\
(2006)[20]\end{array}$ & $\begin{array}{l}N=495, \text { female, } \\
\text { age } 13-18\end{array}$ & $\begin{array}{l}\text { Cross } \\
\text { sectional }\end{array}$ & EAT 40 & $\begin{array}{l}\text { Thin ideal: } 66 \% \text { preferred a } \\
\text { slimmer body than their actual } \\
\text { body. Desire to be thin } \\
\text { associated with elevated EAT } \\
40 \text { scores }\end{array}$ & $p<0.0001$ \\
\hline Country & $\begin{array}{l}\text { Authors } \\
\text { (year) }\end{array}$ & Participants & $\begin{array}{l}\text { Study } \\
\text { design }\end{array}$ & Measures & $\begin{array}{l}\text { Eating disorder-related } \\
\text { variables }\end{array}$ & M (SD)/OR [95\% C.I.]/ r \\
\hline UAE & $\begin{array}{l}\text { O'Hara et al., } \\
(2016)[74]\end{array}$ & $\begin{array}{l}N=420, \text { female, } \\
\text { mean age = } \\
\text { 23.12, university } \\
\text { students }\end{array}$ & $\begin{array}{l}\text { Cross } \\
\text { sectional }\end{array}$ & $\begin{array}{l}\text { EAT } 26 \\
\text { Teasing frequency } \\
\text { from Project eating } \\
\text { attitudes and teens } \\
\text { Weight and body } \\
\text { related shame and } \\
\text { guilt scale }\end{array}$ & $\begin{array}{l}\text { Dieting associated with body } \\
\text { dissatisfaction }\end{array}$ & $r=0.66, p<0.001$ \\
\hline UAE & $\begin{array}{l}\text { Sawadi, Bener, } \\
\text { \& Darmaki } \\
\text { (2000) [88] }\end{array}$ & $\begin{array}{l}N=540, \text { female, } \\
\text { age } 11-19\end{array}$ & $\begin{array}{l}\text { Cross } \\
\text { sectional }\end{array}$ & $\begin{array}{l}\text { Adolescent dieting } \\
\text { scale }\end{array}$ & $\begin{array}{l}\text { Dieting: } 89.4 \% \text { dieting, } 9.1 \% \\
\text { extreme dieting }\end{array}$ & not reported \\
\hline UAE & $\begin{array}{l}\text { Schulte \& } \\
\text { Thomas } \\
\text { (2013) [89] }\end{array}$ & $\begin{array}{l}N=361,284 \\
\text { female, } 77 \text { male, } \\
\text { age } 11-19, \\
\text { university } \\
\text { students }\end{array}$ & $\begin{array}{l}\text { Cross } \\
\text { sectional }\end{array}$ & EAT 26 & $\begin{array}{l}\text { Body image dissatisfaction: } \\
73 \% \text {, female: } 78 \% \text {, male: } 58 \% \text {, } \\
\text { body dissatisfaction associated } \\
\text { with desire to be thin and } \\
\text { elevated EAT score }\end{array}$ & $\begin{array}{l}\text { Desire to be thin: } \times 2(2)= \\
27.083, p<0.001 \text {, EAT: } t(348) \text {, } \\
p<0.001\end{array}$ \\
\hline UAE & $\begin{array}{l}\text { Schulte (2016) } \\
\text { [90] }\end{array}$ & $\begin{array}{l}N=236, \text { mean } \\
\text { age }=19.78\end{array}$ & $\begin{array}{l}\text { Cross } \\
\text { sectional }\end{array}$ & $\begin{array}{l}\text { Body esteem scale, } \\
\text { emotional eating } \\
\text { scale, Weight and } \\
\text { body related } \\
\text { shame and guilt } \\
\text { scale }\end{array}$ & $\begin{array}{l}\text { Disturbed eating behavior and } \\
\text { body dissatisfaction associated } \\
\text { with binge eating }\end{array}$ & $\begin{array}{l}M(S D) \text { : body related shame }= \\
8.00 \text { ( } 8.00), \text { body related } \\
\text { guilt }=11.50 \text { ( } 9.00) \text {. Associations } \\
\text { with binge eating: disturbed } \\
\text { eating behavior: } p<0.001 \\
\text { body dissatisfaction: } p<0.001\end{array}$ \\
\hline UAE & $\begin{array}{l}\text { Thomas, } \\
\text { Khan, \& } \\
\text { Abdulrahman } \\
\text { (2010) [14] }\end{array}$ & $\begin{array}{l}N=228, \text { female, } \\
\text { mean age }=19.8, \\
\text { university } \\
\text { students }\end{array}$ & $\begin{array}{l}\text { Cross } \\
\text { sectional }\end{array}$ & $\begin{array}{l}\text { EAT } 26 \\
\text { FRS }\end{array}$ & $\begin{array}{l}\text { Body dissatisfaction: } 74.8 \% \\
\text { association between body } \\
\text { image dissatisfaction and } \\
\text { disturbed eating behavior }\end{array}$ & $r=0.27, p=0.01$ \\
\hline
\end{tabular}

Note: BSQ Body Shape Questionnaire, EAT Eating Attitude Test, FRS Figure Rating Scale, EDI 2 DT Eating Disorders Inventory 2 Drive for Thinness Scale

in seven cross sectional studies and compensatory behavior $(n=2)$ in a few studies. All ED symptoms were identified by self-report among adolescents: high school and/or university students.

\section{Binge eating}

Kuwaiti and Egyptian Arabs appeared to be most at risk of binge eating. However, binge eating was only assessed among adolescents, and the findings with regard to gender were inconsistent $[11,29,74,77,84,90,92]$.

\section{Restrained eating behavior}

Off the seven studies included in this synthesis that studied restrained eating behavior, all of them reported restrained eating behavior in about one-third of Arabic females. Arab adolescents who reported emotional eating and Arabs with obesity were particularly at risk of restrained eating behavior [20, 23, 29, 66, 67, 77, 87].

\section{Compensatory behavior}

Around $7.5 \%$ of female Arab adolescents reported usage of laxatives [70, 93].

\section{Eating disorders}

Table 3 provides a summary of 27 studies that examined prevalence estimates of Arabs at high risk for EDs. EDscreening instruments were conducted among both males and females, and among adolescents and adults. Arabs at high risk for anorexia nervosa (AN) and bulimia nervosa $(\mathrm{BN})$ were identified in different countries by seven cross-sectional studies. Only one study reported Arabs at high risk for binge eating disorder (BED) and other specified feeding or eating disorders (OSFED) [90]. While most ED assessment tools were self-report measures, in one cross sectional study participants were interviewed using a self-developed interview [39]. Another study used the Structured Clinical Interview for DSM-IV (SCID) [76]. In one case control study among health care clinic attendees, EDs were not 
Table 2 Summary of studies with focus on eating disorder symptoms in the Arab world

\begin{tabular}{|c|c|c|c|c|c|}
\hline $\begin{array}{l}\text { Country/ } \\
\text { Population }\end{array}$ & Authors (year) & Participants & $\begin{array}{l}\text { Study } \\
\text { design }\end{array}$ & Measures & $\begin{array}{l}\text { Eating disorder } \\
\text { symptoms }\end{array}$ \\
\hline Egypt & $\begin{array}{l}\text { Dolan \& Ford (1991) } \\
\text { [11] }\end{array}$ & $N=218$, mean age $=20$, university students & $\begin{array}{l}\text { Cross } \\
\text { sectional }\end{array}$ & $\begin{array}{l}\text { BSQ } \\
\text { Restraint scale } \\
\text { Binge scale }\end{array}$ & $\begin{array}{l}\text { Binge eating: female } 82 \% \text {, } \\
\text { male } 76 \%\end{array}$ \\
\hline Jordan & $\begin{array}{l}\text { Mousa, Mashal, Al- } \\
\text { Domi, \& Jibril (2010) } \\
\text { [84] }\end{array}$ & $N=326$, female, age: $10-16$ & $\begin{array}{l}\text { Cross } \\
\text { sectional }\end{array}$ & $\begin{array}{l}\text { EAT } 26 \\
\text { BSQ }\end{array}$ & Binge eating: $33 \%$ \\
\hline Jordan & $\begin{array}{l}\text { Mousa, Al-Domi, } \\
\text { Mashal, \& Jibril } \\
\text { (2010) [77] }\end{array}$ & $N=432$, female, age: $10-16$ & $\begin{array}{l}\text { Cross } \\
\text { sectional }\end{array}$ & $\begin{array}{l}\text { EAT } 26 \\
\text { BSQ } \\
\text { Eating habits } \\
\text { questionnaire }\end{array}$ & $\begin{array}{l}\text { Binge eating: } 16.9 \% \text {, self- } \\
\text { induced vomiting: } 11 \%\end{array}$ \\
\hline Kuwait & $\begin{array}{l}\text { El-Ghazali, Ibrahim, } \\
\text { Kanari, \& Ismail } \\
\text { (2010) [92] }\end{array}$ & $\begin{array}{l}N=320,223 \text { male, } 97 \text { female, mean age }= \\
21.1\end{array}$ & $\begin{array}{l}\text { Cross } \\
\text { sectional }\end{array}$ & $\begin{array}{l}\text { Questionnaire for } \\
\text { emotional eating } \\
\text { (self- developed) }\end{array}$ & $\begin{array}{l}\text { Emotional eating: female: } \\
85.6 \% \text {, male: } 87.9 \%\end{array}$ \\
\hline Lebanon & $\begin{array}{l}\text { Yahia, El- Ghazale, } \\
\text { Achkar, \& Rizk } \\
\text { (2011) [93] }\end{array}$ & $N=252$, female, students & $\begin{array}{l}\text { Cross } \\
\text { sectional }\end{array}$ & BSQ & Laxatives: $8 \%$, diet pills: $4 \%$ \\
\hline Lebanon & $\begin{array}{l}\text { Zeeni, Gharibeh, \& } \\
\text { Katsounari (2013) } \\
\text { [23] }\end{array}$ & $\begin{array}{l}N=400, \text { female, university students in Cyprus } \\
(n=200) \text { and Lebanon }(n=200)\end{array}$ & $\begin{array}{l}\text { Cross } \\
\text { sectional }\end{array}$ & $\begin{array}{l}\text { Dutch eating } \\
\text { behavior } \\
\text { questionnaire }\end{array}$ & $\begin{array}{l}\text { Restrained eating Lebanese } \\
\text { students: } 30 \%\end{array}$ \\
\hline Jordan & $\begin{array}{l}\text { Afifi- Soweid, } \\
\text { Najem Kteily, \& } \\
\text { Shedia- Rizkallah } \\
\text { (2001) [94] }\end{array}$ & $N=954$, mean age $=18$, university students & $\begin{array}{l}\text { Cross } \\
\text { sectional }\end{array}$ & $\begin{array}{l}\text { Self-developed } \\
\text { questionnaire }\end{array}$ & $\begin{array}{l}\text { Binge eating: } 4.9 \% \text {, secretly } \\
\text { overeating: } 3.7 \%\end{array}$ \\
\hline Jordan & $\begin{array}{l}\text { Mousa, Mashal, Al- } \\
\text { Domi, \& Jibril (2010) } \\
\text { [84] }\end{array}$ & $N=326$, female, age: $10-16$ & $\begin{array}{l}\text { Cross } \\
\text { sectional }\end{array}$ & EAT 26 & Restrained: $40.5 \%$ \\
\hline Oman & $\begin{array}{l}\text { Al Adawi et al., } \\
\text { (2002) [29] }\end{array}$ & $\begin{array}{l}N=293,106 \text { teenagers, mean age }=15.12, \\
100 \text { adults, mean age }=38.71,87 \text { Western } \\
\text { teenagers resided in Oman, mean age }=15.10\end{array}$ & $\begin{array}{l}\text { Cross } \\
\text { sectional }\end{array}$ & $\begin{array}{l}\text { EAT } \\
\text { Bulimic } \\
\text { Investigatory Test }\end{array}$ & $\begin{array}{l}\text { Binge eating: Omani } \\
\text { teenagers 14\%, Western } \\
\text { teenagers 18\% } \\
\text { Restrained: 33\% Omani } \\
\text { teenagers, } 9 \% \text { Western } \\
\text { teenagers }\end{array}$ \\
\hline $\begin{array}{l}\text { Palestinians } \\
\text { residing in the } \\
\text { Northern and } \\
\text { Haifa district }\end{array}$ & $\begin{array}{l}\text { Latzer, Azaiza, \& } \\
\text { Tzischinsky (2009) } \\
{[70]}\end{array}$ & $\begin{array}{l}N=928, \text { female, age: } 12-18,81.2 \% \text { Islamic, } \\
11.2 \% \text { Christian, } 7.6 \% \text { Druze }\end{array}$ & $\begin{array}{l}\text { Cross } \\
\text { sectional }\end{array}$ & EAT 26 & $\begin{array}{l}\text { Laxatives: } 7 \% \text {, self-induced } \\
\text { vomiting: } 8 \%\end{array}$ \\
\hline UAE & Schulte (2016) [90] & $\begin{array}{l}N=236 \\
\text { Mean age }=19.78\end{array}$ & $\begin{array}{l}\text { Cross } \\
\text { sectional }\end{array}$ & $\begin{array}{l}\text { Body esteem } \\
\text { scale } \\
\text { Emotional eating } \\
\text { scale } \\
\text { Weight and body } \\
\text { related shame } \\
\text { and guilt scale }\end{array}$ & $\begin{array}{l}\text { Body esteem scale: } 24.4- \\
35.6 \% \\
\text { Binge eating: moderate } \\
\text { binge eating } 16.9-24.9 \% \text {, } \\
\text { severe binge eating } 6.4- \\
13.2 \% \text {, } \\
\text { Emotional eating scale: } \\
\text { emotional eating } 23-24.5 \%\end{array}$ \\
\hline
\end{tabular}

assessed [69]. Scores above a clinical cut-off on EDscreening instruments were reported without classification specification in 18 studies (Table 3).

\section{Arabs at high risk for ED}

In general, $13-55 \%$ was at high risk for EDs. On EDscreening instruments, females (11.4-54.8\%) displayed more to be at high risk for EDs than males $(2-47.3 \%)$ $[16,18,20,26,28,29,36,39,40,52-55,58,64,66,67$, $69,70,74,76,77,79,80,84,87,89,91]$. Arabs from the UAE appeared to be more at risk for the development of EDs than Arabs from other countries [12, 29, 76, 77].

\section{Correlates}

Table 4 presents a summary of 32 studies on correlates of a high risk for EDs, ED symptoms and ED-related variables. In Western countries, increased affluence and obesity appear to be the major correlates [44]. These factors may also play a role in Arab communities [76], as the Arab world experiences Western and media influences (social media, TV, western advertising and magazines) [84], which may be correlates of the development of EDs [23]. Increased affluence was identified in five cross sectional studies, Western influences in 11 cross sectional studies, media use in five cross sectional 
Table 3 Summary of studies reporting the prevalence of Arabs at high risk for eating disorders

\begin{tabular}{lllll}
\hline $\begin{array}{l}\text { Country/ } \\
\text { Population }\end{array}$ & $\begin{array}{l}\text { Authors } \\
\text { (year) }\end{array}$ & Participants & $\begin{array}{l}\text { Study } \\
\text { design }\end{array}$ & $\begin{array}{l}\text { Measures| } \\
\text { Norms }\end{array}$ \\
\hline $\begin{array}{l}\text { Algeria, Jordan, } \\
\text { Kuwait, Libya, }\end{array}$ & $\begin{array}{l}\text { Musaiger } \\
\text { et al., (2013) }\end{array}$ & $N=4698,2240$ male, 2458 female, age & $\begin{array}{l}\text { Cross } \\
\text { sectional }\end{array}$ & $\begin{array}{l}\text { EAT 26| Saudi } \\
\text { norms }\end{array}$ \\
$\begin{array}{ll}\text { Palestinians residing } \\
\text { in al-Khalil, Syria, UAE }\end{array}$ & [26] & & &
\end{tabular}

Elevated scores at eating disorder screeners $M(S D)$

Above clinical cut off: male:13.847.3\%, female: $16.2-42.7 \%$ Algeria: 15.2\%, male 13.8, female $16.2 \%$

Jordan: $31.6 \%$, male $20.1 \%$,

female $42.7 \%$

Kuwait: $44.7 \%$, male $47.3 \%$,

female $42.8 \%$

Libya: $26.7 \%$, male $19.3 \%$, female $32.6 \%$

Palestinians residing in al-Khalil:

$31.7 \%$, male $23.2 \%$, female $38.9 \%$ Syria: $22.9 \%$, male $14.6 \%$, female $32 \%$

UAE: $33.5 \%$, male $29.8 \%$ female $37.4 \%$

$M(S D)$ not reported

\begin{tabular}{|c|c|c|c|c|c|}
\hline Egypt & $\begin{array}{l}\text { Eladawi et al., } \\
\text { (2018) [28] }\end{array}$ & $\begin{array}{l}N=400,112 \text { male, } 288 \text { female, weight } \\
\text { control center visitors }\end{array}$ & $\begin{array}{l}\text { Cross } \\
\text { sectional }\end{array}$ & $\begin{array}{l}\text { EAT } 40 \mid \text { Western } \\
\text { norms }\end{array}$ & $\begin{array}{l}65 \% \text { above clinical cut off } \\
M(S D)=45.2(10.2) \text { (age } 25-66)\end{array}$ \\
\hline Egypt & $\begin{array}{l}\text { Nasser (1994) } \\
\text { [39] }\end{array}$ & $\begin{array}{l}N=35, \text { female, age } 15-16, \text { secondary } \\
\text { school students }\end{array}$ & $\begin{array}{l}\text { Cross } \\
\text { sectional }\end{array}$ & $\begin{array}{l}\text { EAT } 40 \mid \text { Western } \\
\text { norms } \\
\text { Eating interview }\end{array}$ & $\begin{array}{l}\text { EAT } 40: 11.4 \% \text { above clinical cut } \\
\text { off } \\
M \text { (SD) total sample not reported } \\
\text { Eating interview: } 1.2 \% \text { BN, } 3.4 \% \\
\text { subclinical BN }\end{array}$ \\
\hline Iran & $\begin{array}{l}\text { Abdollahi \& } \\
\text { Mann (2001) } \\
\text { [64] }\end{array}$ & $\begin{array}{l}N=114 \text {, female, university students, } \\
\text { Iranian nationals, } 45 \text { resided in LA, } 59 \\
\text { resided in Teheran }\end{array}$ & $\begin{array}{l}\text { Cross } \\
\text { sectional }\end{array}$ & $\begin{array}{l}\text { EDE-Q } \\
\text { FRS }\end{array}$ & $\begin{array}{l}\% \text { above clinical cutoff not } \\
\text { reported }\end{array}$ \\
\hline Iran & $\begin{array}{l}\text { Raouf et al., } \\
\text { (2015) [76] }\end{array}$ & $\begin{array}{l}N=1990,951 \text { male, } 1039 \text { female, age } \\
13-18, \text { mean age }=15.8\end{array}$ & $\begin{array}{l}\text { Cross } \\
\text { sectional }\end{array}$ & $\begin{array}{l}\text { EAT 26| Irani } \\
\text { norms } \\
\text { SCID }\end{array}$ & $\begin{array}{l}\text { EAT 26: } 24.2 \% \text { above clinical cut } \\
\text { off } \\
M \text { (SD) not reported } \\
\text { SCID: } 0.25 \% \text { diagnosed with ED, } \\
0.7 \% \text { AN, } 0.9 \% \text { BN, } 1.0 \% \text { OSFED }\end{array}$ \\
\hline Jordan & $\begin{array}{l}\text { Madanat et al., } \\
\text { (2007) [80] }\end{array}$ & $N=800$, female, mean age $=33.5$ & $\begin{array}{l}\text { Cross } \\
\text { sectional }\end{array}$ & $\begin{array}{l}\text { EAT 26| Saudi } \\
\text { norms } \\
\text { Motivation for } \\
\text { eating scale } \\
\text { Restraint scale } \\
\text { Sociocultural } \\
\text { attitudes towards } \\
\text { appearance scale } \\
\text { Body esteem } \\
\text { scale } \\
\text { 9- figure } \\
\text { silhouettes }\end{array}$ & $\begin{array}{l}\text { EAT 26: } 54.8 \% \text { above clinical cut } \\
\text { off } \\
M(S D)=18.98(10.76)\end{array}$ \\
\hline Jordan & $\begin{array}{l}\text { Mousa, } \\
\text { Mashal, Al- } \\
\text { Domi, \& Jibril } \\
\text { (2010) [84] }\end{array}$ & $N=326$, female, age: $10-16$ & $\begin{array}{l}\text { Cross } \\
\text { sectional }\end{array}$ & $\begin{array}{l}\text { EAT 26| Western } \\
\text { norms } \\
\text { BSQ| Kuwaiti } \\
\text { norms }\end{array}$ & $\begin{array}{l}\text { EAT 26: } 40.5 \% \text { above clinical cut } \\
\text { off } \\
M(S D)=16.6(10.7)\end{array}$ \\
\hline Jordan & $\begin{array}{l}\text { Mousa, Al- } \\
\text { Domi, et al., } \\
(2010)[77]\end{array}$ & $N=432$, female, age: $10-16$ & $\begin{array}{l}\text { Cross } \\
\text { sectional }\end{array}$ & $\begin{array}{l}\text { Eating habits } \\
\text { questionnaire }\end{array}$ & $\begin{array}{l}\text { OSFED: } 31 \%, \text { BED: } 1.8 \%, \text { BN: } 0.6 \% \text {, } \\
\text { AN: } 0 \% \\
M \text { (SD) not reported }\end{array}$ \\
\hline Kuwait & $\begin{array}{l}\text { Alkhadari } \\
\text { et al., (2016) } \\
\text { [69] }\end{array}$ & $\begin{array}{l}N=1046,429 \text { male, } 617 \text { female, mean } \\
\text { age }=37.6, \text { health care clinic attendees }\end{array}$ & $\begin{array}{l}\text { Case control } \\
\text { (health care } \\
\text { clinic } \\
\text { attendees) }\end{array}$ & $\begin{array}{l}\text { Patient health } \\
\text { questionnaires } \\
\text { GAD-7 }\end{array}$ & $\begin{array}{l}\text { Eating disorders were not } \\
\text { assessed }\end{array}$ \\
\hline Kuwait & $\begin{array}{l}\text { Ebrahim, } \\
\text { Alkazemi, } \\
\text { Zafar, \& } \\
\text { Kubow (2019) } \\
{[86]}\end{array}$ & $N=400$, male, university students & $\begin{array}{l}\text { Cross } \\
\text { sectional }\end{array}$ & $\begin{array}{l}\text { EAT 26| Saudi } \\
\text { norms }\end{array}$ & $\begin{array}{l}46.2 \% \text { above clinical cut off } \\
M(S D)=20.4(14.1)\end{array}$ \\
\hline Lebanon & $\begin{array}{l}\text { Aoun et al., } \\
\text { (2015) [54] }\end{array}$ & $\begin{array}{l}N=123 \text {, female, age } 15-55 \text {, primary } \\
\text { health care center visitors }\end{array}$ & $\begin{array}{l}\text { Validation } \\
\text { SCOFF }\end{array}$ & SCOFF & $\begin{array}{l}28 \% \text { above clinical cut off } \\
M(S D) \text { not reported }\end{array}$ \\
\hline
\end{tabular}


Table 3 Summary of studies reporting the prevalence of Arabs at high risk for eating disorders (Continued)

\begin{tabular}{|c|c|c|c|c|c|}
\hline $\begin{array}{l}\text { Country/ } \\
\text { Population }\end{array}$ & $\begin{array}{l}\text { Authors } \\
\text { (year) }\end{array}$ & Participants & $\begin{array}{l}\text { Study } \\
\text { design }\end{array}$ & $\begin{array}{l}\text { Measures| } \\
\text { Norms }\end{array}$ & $\begin{array}{l}\text { Elevated scores at eating } \\
\text { disorder screeners } M(S D)\end{array}$ \\
\hline Oman & $\begin{array}{l}\text { Kayano et al., } \\
\text { (2008), Viernes } \\
\text { et al., (2007) } \\
{[52,58]}\end{array}$ & $\begin{array}{l}N=248,135 \text { Omani, } 113 \text { Westerners } \\
\text { resided in Oman, age 13-18 }\end{array}$ & $\begin{array}{l}\text { Cross } \\
\text { sectional }\end{array}$ & $\begin{array}{l}\text { EAT 26| Saudi } \\
\text { norms } \\
\text { EDI } 2 \text { DT| Saudi } \\
\text { norms }\end{array}$ & $\begin{array}{l}\text { \% above cut-off not reported } \\
\text { EAT: Omani: } M(S D)=8.48(1.64) \text {, } \\
\text { European expatriates: } M(S D)= \\
5.98 \text { (1.83) } \\
\text { EDI2 DT: Omani: } M(S D)=4.12 \\
(0.60) \text {, European expatriates: } M \\
(S D)=10.14(0.64) .\end{array}$ \\
\hline Country & $\begin{array}{l}\text { Authors } \\
\text { (year) }\end{array}$ & Participants & $\begin{array}{l}\text { Study } \\
\text { design }\end{array}$ & $\begin{array}{l}\text { Measures } \\
\text { Norms }\end{array}$ & $\begin{array}{l}\text { Elevated scores at eating } \\
\text { disorder screeners } M(S D)\end{array}$ \\
\hline Oman & $\begin{array}{l}\text { Al Adawi et al., } \\
\text { (2002) [29] }\end{array}$ & $\begin{array}{l}N=293,106 \text { teenagers, mean age }= \\
15.12,100 \text { adults, mean age }=38.71 ; 87 \\
\text { Western teenagers resided in Oman, } \\
\text { mean age }=15.10\end{array}$ & $\begin{array}{l}\text { Cross } \\
\text { sectional }\end{array}$ & $\begin{array}{l}\text { EAT 26| Saudi } \\
\text { norms } \\
\text { Bulimic } \\
\text { Investigatory Test }\end{array}$ & $\begin{array}{l}\text { EAT: } 33 \% \text { Omani teenagers, } 9 \% \\
\text { Western teenagers above clinical } \\
\text { cut off } \\
M \text { (SD) not reported } \\
\text { Bulimic Investigatory Test: } 12.3 \% \\
\text { Omani teenagers, } 2 \% \text { Omani } \\
\text { adults, } 18.4 \% \text { Western teenagers }\end{array}$ \\
\hline $\begin{array}{l}\text { Palestinians residing } \\
\text { in the Northern and } \\
\text { Haifa district }\end{array}$ & $\begin{array}{l}\text { Latzer et al., } \\
\text { (2009) [70] }\end{array}$ & $\begin{array}{l}N=1141, \text { female, age: } 12-18,81.2 \% \\
\text { Islamic, } 11.2 \% \text { Christian, } 7.6 \% \text { Druze }\end{array}$ & $\begin{array}{l}\text { Cross } \\
\text { sectional }\end{array}$ & $\begin{array}{l}\text { EAT 26| Saudi } \\
\text { norms }\end{array}$ & $\begin{array}{l}25 \% \text { above clinical cut off } \\
M(S D) \text { age } 12-13=16.5(11.9) \\
\text { age } 14-15=15.0(10.5), \text { age } 16- \\
18=15.2(9.7)\end{array}$ \\
\hline $\begin{array}{l}\text { Palestinians residing } \\
\text { in Nablus }\end{array}$ & $\begin{array}{l}\text { Saleh et al., } \\
\text { (2018) [36] }\end{array}$ & $N=2001$, female university students & $\begin{array}{l}\text { Cross } \\
\text { sectional }\end{array}$ & $\begin{array}{l}\text { EAT 26| Saudi } \\
\text { norms } \\
\text { SCOFF }\end{array}$ & $\begin{array}{l}\text { EAT: } 28.6 \% \text { above clinical cut off } \\
M(S D)=15.27 \text { (10.38) } \\
\text { SCOFF: } 38.2 \% \text { above clinical cut } \\
\text { off } \\
M(S D)=1.25(1.032)\end{array}$ \\
\hline Qatar and Lebanon & $\begin{array}{l}\text { Kronfol et al., } \\
\text { (2018) [55] }\end{array}$ & $\begin{array}{l}N=1841,167 \text { Lebanon, } 785 \text { Qatar, } 889 \\
\text { USA university students }\end{array}$ & $\begin{array}{l}\text { Cross } \\
\text { sectional }\end{array}$ & SCOFF & $\begin{array}{l}\text { Arab students: } 20.4 \% \text { above } \\
\text { clinical cut off } \\
\text { American students: } 6.8 \% \text { above } \\
\text { clinical cut off } \\
M(S D) \text { not reported }\end{array}$ \\
\hline Saudi Arabia & $\begin{array}{l}\text { Al- Subaie } \\
(2000)[87]\end{array}$ & $N=1179$, female, mean age $=16.1$ & $\begin{array}{l}\text { Cross } \\
\text { sectional }\end{array}$ & EDI 2 DT & $\begin{array}{l}\text { 15.9\% above clinical cut off } \\
\text { M } 6.7 \text { SD not reported }\end{array}$ \\
\hline Saudi Arabia & $\begin{array}{l}\text { Bano et al., } \\
\text { (2013) [67] }\end{array}$ & $N=100$, female, age $18-25$ & $\begin{array}{l}\text { Cross } \\
\text { sectional }\end{array}$ & $\begin{array}{l}\text { EAT 26| Western } \\
\text { norms }\end{array}$ & $\begin{array}{l}\text { Female: } 24 \% \text { above clinical cut } \\
\text { off, male: } 2 \% \text { above clinical cut } \\
\text { off } \\
M \text { (SD) female }=16.89(10.52), M \\
\text { (SD) male }=9.88(13.26)\end{array}$ \\
\hline Saudi Arabia & $\begin{array}{l}\text { Fallatah et al., } \\
\text { (2015) [66] }\end{array}$ & $N=425$, female, age $15-18$ & $\begin{array}{l}\text { Cross } \\
\text { sectional }\end{array}$ & $\begin{array}{l}\text { EAT 26| Saudi } \\
\text { norms }\end{array}$ & $\begin{array}{l}\text { 32.9\% above clinical cut off } \\
M(S D)=17.98(9.29)\end{array}$ \\
\hline UAE & $\begin{array}{l}\text { Eapen et al., } \\
\text { (2006) [20] }\end{array}$ & $N=495$, female, age 13-18 & $\begin{array}{l}\text { Cross } \\
\text { sectional }\end{array}$ & $\begin{array}{l}\text { EAT 40| Western } \\
\text { norms }\end{array}$ & $\begin{array}{l}23.4 \% \text { above clinical cut off } \\
M(S D)=15.19(1.94)\end{array}$ \\
\hline UAE & $\begin{array}{l}\text { Musaiger, Al- } \\
\text { Mannai, \& Al- } \\
\text { Lalla (2014) } \\
{[79]}\end{array}$ & $\begin{array}{l}N=731 \text {, male, age } 15-18, \text { resided in } 5 \\
\text { different Emirates }\end{array}$ & $\begin{array}{l}\text { Cross } \\
\text { sectional }\end{array}$ & $\begin{array}{l}\text { EAT 26| Saudi } \\
\text { norms }\end{array}$ & $\begin{array}{l}\text { \% above clinical cut off: Dubai } \\
49.1 \% \text {, Ajman } 33.1 \% \text {, Al Fujairah } \\
\text { 48.0\%, Ras al Khaima 34.8\%, Um } \\
\text { al Quain } 39.7 \% \\
\text { M (SD) not reported }\end{array}$ \\
\hline UAE & $\begin{array}{l}\text { O'Hara et al., } \\
\text { (2016) [74] }\end{array}$ & $\begin{array}{l}N=420, \text { female, mean age }=23.12, \\
\text { university students }\end{array}$ & $\begin{array}{l}\text { Cross } \\
\text { sectional }\end{array}$ & $\begin{array}{l}\text { EAT 26| Western } \\
\text { norms } \\
\text { Teasing } \\
\text { frequency from } \\
\text { Project eating } \\
\text { attitudes and } \\
\text { teens } \\
\text { Weight and body } \\
\text { related shame } \\
\text { and guilt scale }\end{array}$ & $\begin{array}{l}\text { EAT 26: } 30 \% \text { above clinical cut off } \\
M(S D)=15.57(9.03)\end{array}$ \\
\hline UAE & $\begin{array}{l}\text { Schulte \& } \\
\text { Thomas (2013) } \\
\text { [89] }\end{array}$ & $\begin{array}{l}N=361,77 \text { male, } 284 \text { female, age } 11- \\
19\end{array}$ & $\begin{array}{l}\text { Cross } \\
\text { sectional }\end{array}$ & $\begin{array}{l}\text { EAT 26| Western } \\
\text { norms }\end{array}$ & $\begin{array}{l}20 \% \text { above clinical cut off } \\
M \text { (SD) female }=12.88(8.91), M \\
\text { (SD) male }=11.21(9.81)\end{array}$ \\
\hline
\end{tabular}


Table 3 Summary of studies reporting the prevalence of Arabs at high risk for eating disorders (Continued)

\begin{tabular}{|c|c|c|c|c|c|}
\hline $\begin{array}{l}\text { Country/ } \\
\text { Population }\end{array}$ & $\begin{array}{l}\text { Authors } \\
\text { (year) }\end{array}$ & Participants & $\begin{array}{l}\text { Study } \\
\text { design }\end{array}$ & $\begin{array}{l}\text { Measures| } \\
\text { Norms }\end{array}$ & $\begin{array}{l}\text { Elevated scores at eating } \\
\text { disorder screeners } M(S D)\end{array}$ \\
\hline UAE & $\begin{array}{l}\text { Thomas et al., } \\
\text { (2010) [14] }\end{array}$ & $\begin{array}{l}N=228, \text { female, } \text { mean age }=19.8, \\
\text { university students }\end{array}$ & $\begin{array}{l}\text { Cross } \\
\text { sectional }\end{array}$ & $\begin{array}{l}\text { EAT 26| Western } \\
\text { norms }\end{array}$ & $\begin{array}{l}24.6 \% \text { above clinical cut off } \\
M(S D)=13.31(10.21)\end{array}$ \\
\hline UAE & $\begin{array}{l}\text { Thomas, } \\
\text { O'Hara, et al., } \\
\text { (2018) [53] }\end{array}$ & $N=1069$, female, university students & $\begin{array}{l}\text { Cross } \\
\text { sectional }\end{array}$ & $\begin{array}{l}\text { EAT 26| Western } \\
\text { norms }\end{array}$ & $\begin{array}{l}29.0 \% \text { above clinical cut off } \\
M(S D)=15.80(9.39)\end{array}$ \\
\hline UAE & $\begin{array}{l}\text { Thomas, } \\
\text { O'Hara, et al., } \\
\text { (2018) [18] }\end{array}$ & $N=209$, female, university students & $\begin{array}{l}\text { Cross } \\
\text { sectional }\end{array}$ & $\begin{array}{l}\text { EAT 26| Western } \\
\text { norms }\end{array}$ & $\begin{array}{l}\text { 30.3\% above clinical cut off } \\
M(S D)=14.17(9.40)\end{array}$ \\
\hline
\end{tabular}

Note: BSQ Body Shape Questionnaire, EAT Eating Attitude Test, EDE-Q Eating Disorder Examination Questionnaire, FRS Figure Rating Scale, EDI 2 DT Eating Disorders Inventory 2 Drive for Thinness Scale, SCID Structured Clinical Interview for DSM IV, SCOFF Sick, Control, One, Stone, Fat, Food

studies, and obesity in nine cross-sectional studies. Most were identified by self-report. However, one study investigated the association between media use and EDs in several countries with a self-developed interview [27], and another study examined EDs with the SCID [76].

\section{Increased affluence}

Increased affluence was associated with ED-related variables $[16,90,96]$, especially among Saudis $[24,62,69,90]$.

\section{Western influences}

Exposure to Western influences was associated with EDrelated variables, especially the desire to be thin $[13,22$, 37, 70]. Examples of such Western influences were media [14, 31], traveling abroad [35], living abroad [90, 92, 97], and contact with expatriates [13, 18, 35, 37]. Arabs with greater assimilation with the Western culture were potentially at more at risk for EDs [22]. Arabs in Gulf countries (Oman, Saudi Arabia, UAE) were particularly vulnerable to Western influences. However, besides Western influences vulnerability for EDs might also be associated with industrialization, as the Gulf also has the highest level of industrialization within the Arab world $[13,37]$.

\section{Media use}

Usage of social media was associated with ED symptoms [98, 99], greater body dissatisfaction, the desire to be thin [100-102], and disturbed eating behavior [94, 103]. Media use was a particular risk factor among adolescents $[18,23,69,74,83]$.

\section{Obesity}

A higher BMI was positively associated with ED-related variables [74, 104-106], ED symptoms [24, 62] and high risk for EDs [22]. This is of concern because the Arab world has one of the highest rates of obesity in the world [107], in Saudi Arabia in particular [108], followed by Bahrain, Egypt, Jordan, Syria and Oman [91].

\section{Cultural differences in eating disorders}

Some studies suggest that, rather than causing a rise in EDs, Western influences are associated with a shift in symptomatology. The four cross-sectional studies summarized in Table 4 examined cultural differences in EDs.

\section{Cultural differences}

Interestingly, predominant identification with a Middle Eastern culture was associated with a risk for AN [29, 64], while predominant identification with a Western culture was associated with a risk for BN [64], for instance among European expatriates resident in Oman, among Omani Arabs [29] and among Iranians [64]. Omani Arabs tended to express their ED symptoms somatically (e.g. bloated stomach, nausea, reduced, fluttering feeling in the stomach, throat discomfort, etc.), while Western populations were more likely to express them psychiatrically $[52,58]$.

\section{Discussion}

The aim of this study was to provide estimates regarding the point-prevalence of Arabs at high risk for EDs, with ED symptoms and ED-related variables. An additional aim was to provide a comprehensive synthesis of relevant studies of correlates and ED-related variables. Although EDs occur both in Western [60] and in Arab societies [76], there are no official statistics available for in the Arab world [96] and prevalence has rarely been studied [90]. The prevalence of full syndrome EDs have not been reported in the Arab world, except for one case study [97]. Perhaps this is because EDs are not viewed as common disorders in the Arab world [69]. This synthesis found that in the Arab world, $13-55 \%$ is at high risk for EDs, the prevalence was higher among females than in males $[16,18,20,26,28,29,36,39,40,52-55,58,64$, $66,67,69,70,74,76,77,79,80,84,87,89,91]$, and the prevalence appeared to increase during adolescence $[20$, $76,98,99]$. There were also some indications symptomatology was culturally reactive $[12,29,52,58,64,87]$.

EDs are perceived as diseases of globalization [6]. The rapid sociocultural changes in the Arab world [20] since 
Table 4 Summary of studies reporting correlates of eating disorders and eating disorder-related variables in the Arab world

\begin{tabular}{|c|c|c|c|c|c|}
\hline $\begin{array}{l}\text { Country/ } \\
\text { Population }\end{array}$ & $\begin{array}{l}\text { Authors } \\
\text { (year) }\end{array}$ & Participants & $\begin{array}{l}\text { Study } \\
\text { design }\end{array}$ & Measures & Risk factors \\
\hline $\begin{array}{l}\text { Algeria, } \\
\text { Jordan, } \\
\text { Kuwait, Libya, } \\
\text { Palestinians } \\
\text { residing in al- } \\
\text { Khalil, Syria, } \\
\text { UAE }\end{array}$ & $\begin{array}{l}\text { Musaiger } \\
\text { et al., (2013) } \\
\text { [26] }\end{array}$ & $\begin{array}{l}N=4698,2240 \text { male, } \\
2458 \text { female, age } 15-18\end{array}$ & $\begin{array}{l}\text { Cross } \\
\text { sectional }\end{array}$ & EAT 26 & $\begin{array}{l}\text { Obesity: disturbed eating } \\
\text { behavior } 2-3 \text { times as high } \\
\text { obese than in non-obese } \\
\text { males and females }\end{array}$ \\
\hline $\begin{array}{l}\text { Bahrain, } \\
\text { Egypt, Jordan, } \\
\text { Oman, Syria }\end{array}$ & $\begin{array}{l}\text { Musaiger } \\
\text { (2014) [27] }\end{array}$ & $\begin{array}{l}N=1134, \text { female, } \\
\text { university students, age } \\
17-32\end{array}$ & $\begin{array}{l}\text { Cross } \\
\text { sectional }\end{array}$ & $\begin{array}{l}\text { Interview (self- } \\
\text { developed) }\end{array}$ & $\begin{array}{l}\text { Media use: exposure to } \\
\text { magazines associated with } \\
\text { dieting to lose weight in } \\
\text { Bahrain, exposure to TV } \\
\text { associated with desire to be } \\
\text { thin in Egypt, Oman and } \\
\text { Jordan, exposure to TV } \\
\text { associated with dieting to } \\
\text { lose weight in Egypt and } \\
\text { Bahrain }\end{array}$ \\
\hline
\end{tabular}

\begin{tabular}{|c|c|c|c|c|}
\hline Egypt & $\begin{array}{l}\text { Eladawi et al., } \\
\text { (2018) [28] }\end{array}$ & $\begin{array}{l}N=400,112 \text { male, } 288 \\
\text { female, weight control } \\
\text { center visitors }\end{array}$ & $\begin{array}{l}\text { Case } \\
\text { control }\end{array}$ & EAT 40 \\
\hline Iran & $\begin{array}{l}\text { Abdollahi \& } \\
\text { Mann (2001) } \\
\text { [64] }\end{array}$ & $\begin{array}{l}N=114 \text {, female, } \\
\text { university students, } \\
\text { Iranian nationals, } 45 \\
\text { resided in LA, } 59 \text { resided } \\
\text { in Teheran }\end{array}$ & $\begin{array}{l}\text { Cross } \\
\text { sectional }\end{array}$ & EDE-Q \\
\hline Iran & $\begin{array}{l}\text { Raouf et al., } \\
\text { (2015) [76] }\end{array}$ & $\begin{array}{l}N=1990,951 \text { male, } 1039 \\
\text { female, age } 13-18, \text { mean } \\
\text { age }=15.8\end{array}$ & $\begin{array}{l}\text { Cross } \\
\text { sectional }\end{array}$ & $\begin{array}{l}\text { EAT } 26 \\
\text { SCID }\end{array}$ \\
\hline
\end{tabular}

\begin{tabular}{|c|c|c|c|c|}
\hline Jordan & $\begin{array}{l}\text { Madanat } \\
\text { et al., (2007) } \\
\text { [80] }\end{array}$ & $\begin{array}{l}N=800, \text { female, } \text { mean } \\
\text { age }=33.5\end{array}$ & $\begin{array}{l}\text { Cross } \\
\text { sectional }\end{array}$ & $\begin{array}{l}\text { EAT } 26 \\
\text { Motivation for } \\
\text { eating scale } \\
\text { Restraint scale } \\
\text { Sociocultural } \\
\text { attitudes } \\
\text { towards } \\
\text { appearance } \\
\text { scale } \\
\text { Body esteem } \\
\text { scale } \\
\text { 9- figure } \\
\text { silhouettes }\end{array}$ \\
\hline Jordan & $\begin{array}{l}\text { Madanat, } \\
\text { Hawks, \& } \\
\text { Angeles } \\
\text { (2011) [83] }\end{array}$ & $N=800$, female & $\begin{array}{l}\text { Cross } \\
\text { sectional }\end{array}$ & $\begin{array}{l}\text { 9- figure } \\
\text { silhouettes }\end{array}$ \\
\hline Jordan & $\begin{array}{l}\text { Mousa, } \\
\text { Mashal, Al- } \\
\text { Domi, \& Jibril } \\
\text { (2010) [84] }\end{array}$ & $\begin{array}{l}N=326, \text { female, age: } 10- \\
16\end{array}$ & $\begin{array}{l}\text { Cross } \\
\text { sectional }\end{array}$ & $\begin{array}{l}\text { EAT } 26 \\
\text { BSQ }\end{array}$ \\
\hline
\end{tabular}

Weight status: $53.8 \%$ overweight/ obese. Obesity associated with desire to lose weight, restrained eating, emotional eating, elevated EAT scores. Western advertising and media use associated with desire to lose weight, restrained eating, emotional eating, disturbed eating behavior. Increased affluence associated with elevated EAT scores

BMl: $53.8 \%$ overweight/ obese, $66 \%$ body dissatisfaction, association between $\mathrm{BMl}$ and desire to lose weight

Media use associated with body dissatisfaction. BMI associated with body dissatisfaction. Residing in a
Associations: magazines and dieting: Bahrain: $\mathrm{OR}=2.29$ [0.95-5.68], $p<0.044$, Egypt OR=6.29 [2.21-17.39], $p<0.001$, Jordan $\mathrm{OR}=5.29$ [1.78-16.83], $p<0.001$. $\mathrm{TV}$ and desire to be thin: Egypt $\mathrm{OR}=2.05$ [1.07-3.94], $p<0.019$, Oman $p<0.019$,

$\mathrm{OR}=2.41[1.09-5.48]$.

TV and dieting: Bahrain $\mathrm{OR}=$ 1.98 [1.00-3.94], $p<0.035$, Egypt $\mathrm{OR}=2.21[1.01-4.92]$, $p<0.032$

Rural residents: $\mathrm{OR}=1.75$ [0.95-3.22], $p=0.044$, affluence: $\mathrm{OR}=3.17$ [0.7413.63], $p=0.023$, weight: $p=$ 0.006; overweight $\mathrm{OR}=2.75$

[1.42-5.33], obesity $\mathrm{OR}=1.46$ [0.82-2.59]

$p<0.05$

difference between actual and desired BMl larger in LA BMI, age, increased affluence, female gender associated

Female: EAT OR $=2.52[0.42-$ $0.651, p<0.001$, AN $p<0.001$, BN $p<0.05$, OSFED $p<0.001$, age: $\mathrm{OR}=1.09$ [0.99-1.17], $p=0.036, \mathrm{BMI}: \mathrm{OR}=0.93$ [0.90-0.96], $p<0.001$, increased affluence: $\mathrm{OR}=1.17$ [1.01-1.35], $p=0.029$

Obesity: $p<0.01$ Increased affluence: $p<0.01$. Associations Western advertising and media not reported.

$$
r=0.858, p<0.0001
$$

Obesity: $\mathrm{OR}=2.8[2.1-3.8]$, $p<0.01$ Media: $\mathrm{OR}=1.2[1.1-1.4], p<$ 0.01 , reside in Western 
Table 4 Summary of studies reporting correlates of eating disorders and eating disorder-related variables in the Arab world (Continued)

\begin{tabular}{|c|c|c|c|c|c|c|}
\hline $\begin{array}{l}\text { Country/ } \\
\text { Population }\end{array}$ & $\begin{array}{l}\text { Authors } \\
\text { (year) }\end{array}$ & Participants & $\begin{array}{l}\text { Study } \\
\text { design }\end{array}$ & Measures & Risk factors & $M(S D) /$ OR $[95 \% \mathrm{Cl}] / r$ \\
\hline & & & & & $\begin{array}{l}\text { Western country is protective } \\
\text { factor for body dissatisfaction. }\end{array}$ & $\begin{array}{l}\text { country: [RR: } 0.34(0.12-1.1) \\
p=0.046]\end{array}$ \\
\hline Jordan & $\begin{array}{l}\text { Zawawi } \\
\text { (2014) [85] }\end{array}$ & $\begin{array}{l}N=170, \text { female, age: } 20- \\
55, \text { fitness center users }\end{array}$ & $\begin{array}{l}\text { Cross } \\
\text { sectional }\end{array}$ & $\mathrm{BSQ}$ & $\begin{array}{l}\text { BMI: positive association } \\
\text { between BMI and body } \\
\text { dissatisfaction }\end{array}$ & $\begin{array}{l}r=0.729, r^{2}=0.53, F(1,175)= \\
198.6, p<0.01\end{array}$ \\
\hline
\end{tabular}

\begin{tabular}{|c|c|c|}
\hline Country & $\begin{array}{l}\text { Authors } \\
\text { (year) }\end{array}$ & Participants \\
\hline Kuwait & $\begin{array}{l}\text { Ebrahim, } \\
\text { Alkazemi, } \\
\text { Zafar, \& } \\
\text { Kubow (2019) } \\
\text { [86] }\end{array}$ & $\begin{array}{l}N=400, \text { Male, university } \\
\text { students }\end{array}$ \\
\hline Kuwait & $\begin{array}{l}\text { Musaiger \& } \\
\text { Al- Mannai } \\
\text { (2013) [75] }\end{array}$ & $\begin{array}{l}N=228, \text { female, } \\
\text { university students, age } \\
19-25\end{array}$ \\
\hline
\end{tabular}

$\begin{array}{lll}\text { Lebanon and } & \text { Zeeni, } & N=400, \text { female, university } \\ \text { Cyprus } & \text { Gharibeh, \& } & \text { students in Cyprus }(n= \\ & \text { Katsounari } & \text { 200) and Lebanon }(n= \\ & \text { (2013) [23] } & \text { 200) }\end{array}$

\begin{tabular}{|c|c|c|c|c|}
\hline Oman & $\begin{array}{l}\text { Al Adawi } \\
\text { et al., (2002) } \\
\text { [29] }\end{array}$ & $\begin{array}{l}N=293,106 \text { teenagers, } \\
\text { mean age }=15.12,100 \\
\text { adults, mean age }=38.71 ; \\
87 \text { Western teenagers } \\
\text { resided in Oman, mean } \\
\text { age }=15.10\end{array}$ & $\begin{array}{l}\text { Cross } \\
\text { sectional }\end{array}$ & $\begin{array}{l}\text { EAT } \\
\text { Bulimic } \\
\text { Investigatory } \\
\text { Test }\end{array}$ \\
\hline Oman & $\begin{array}{l}\text { Kayano et al., } \\
\text { (2008) [58] }\end{array}$ & $\begin{array}{l}N=248,135 \text { Omani, } 113 \\
\text { Westerners resided in } \\
\text { Oman, age } 13-18\end{array}$ & $\begin{array}{l}\text { Cross } \\
\text { sectional }\end{array}$ & $\begin{array}{l}\text { EAT } 26 \\
\text { EDI } 2 \text { DT }\end{array}$ \\
\hline
\end{tabular}

Oman Viernes et al., $N=248,135$ Omani, 113 Cross EAT 26 (2007) [52] Westerners resided in sectional EDI 2 DT Oman, age 13-18

\section{Study Measures Risk factors design \\ Cross EAT 26 \\ sectional \\ Obesity associated with disordered eating and dieting.}

Cross Questions

sectional validated by Field et al., 2005, translated into Arabic

Cross

Dutch eating

sectional behavior questionnaire Perceived sociocultural influences on body image and body change questionnaire

Use of internet and reading magazines associated with dieting to lose weight, media influence 2-3 times higher in obese than in non-obese females, watching TV not associated with body shape concern.

Lebanese students greater association between body image dissatisfaction and media use. Greater emotional eating and sociocultural influences in eating behavior, greater influence of media to become slimmer, eat less and exercise to lose weight $(p<$

0.05). Association between

$\mathrm{BMI}$ and restraint and emotional eating in Lebanon and in Cyprus. No differences in restraint eating

Westernization: significant difference in BMI between Omani and Western teenagers, Omani teenagers significantly more susceptible for AN and BN than Western teenagers

Weight status: $13 \%$ obese, 27\% underweight. BMI associated with desire to be thin, negative association between EDI and EAT 26 scores. Average score on EAT 26 higher in the Omani (9.2) than in the Western (5.59) group. EDI score 3 times higher in Western than in Omani group.

$\mathrm{BMI}$ associated with desire to be thin and guilt after eating sweets. Terrified to become fat: European expats: 81\%, Omani's: 66\%. higher fear of fatness. Somatic symptom presentation among Omani's.

\section{$M(S D) /$ OR $[95 \% \mathrm{Cl}] / r$}

Disordered eating: $\mathrm{OR}=2.06$ $[1.17,3.60], p=0.011$, Dieting: $\mathrm{OR}=2.063[1.01,4.21], p=$ 0.043)

Dieting: $\mathrm{OR}=3.11$ [1.5-6.47], Media influence: $\mathrm{OR}=2.14$ [0.93-5.09], internet: $p=$ 0.000 , magazines: $p=0.011$, media influence in obese: $p=0.000$

Associations Lebanon: BMI and restrained $r=0.3, p<$ $0.001, \mathrm{BMI}$ and emotional eating $r=0.2, p=0.01$, media to become slimmer $t(371.66)=5.02, p<0.001, r=$ $0.25)$, eat less to lose weight $t(383.31)=3.02, p<0.001, r=$ 0.15 , exercise more to lose weight $t(380.90)=3.53, p<$ $0.001, r=0.18$

BMI: $p=0.000$

BMI: $r=0.03, p<0.05$, Omani higher EAT scores: $F(2,240)=$ $10.95, p<0.001$. Europeans higher EDI 2 DT scores: $F(2$, $240)=71.72, p<0.001$

Desire to be thin: Omani $\mathrm{OR}=1.60$ [0.92 2.79], $p=0.09$, European expats: $\mathrm{OR}=8.17$ [4.63 14.41], $p=0.00$, guilt after eating sweets: Omani $\mathrm{OR}=0.05\left[\begin{array}{ll}0.01 & 0.36\end{array}\right], p=0.00$. Terrified to become fat: $F=$ $235.9, p<0.001$. Somatic symptom presentation: Kendall's tau $=0.352, p<$ 
Table 4 Summary of studies reporting correlates of eating disorders and eating disorder-related variables in the Arab world (Continued)

\begin{tabular}{|c|c|c|c|c|c|c|}
\hline $\begin{array}{l}\text { Country/ } \\
\text { Population }\end{array}$ & $\begin{array}{l}\text { Authors } \\
\text { (year) }\end{array}$ & Participants & $\begin{array}{l}\text { Study } \\
\text { design }\end{array}$ & Measures & Risk factors & $M(S D) /$ OR $[95 \% \mathrm{Cl}] / r$ \\
\hline & & & & & & 0.001 \\
\hline $\begin{array}{l}\text { Palestinians } \\
\text { residing in } \\
\text { the Northern } \\
\text { and Haifa } \\
\text { district }\end{array}$ & $\begin{array}{l}\text { Latzer et al., } \\
\text { (2009) [70] }\end{array}$ & $\begin{array}{l}N=1141, \text { female, age: } \\
12-18,81.2 \% \text { Islamic, } \\
11.2 \% \text { Christian, } 7.6 \% \\
\text { Druze }\end{array}$ & $\begin{array}{l}\text { Cross } \\
\text { sectional }\end{array}$ & EAT 26 & $\begin{array}{l}\text { Westernization: Druze } \\
\text { subgroup higher scores on } \\
\text { EAT }\end{array}$ & $F[2]=2.9, p<0.05$ \\
\hline $\begin{array}{l}\text { Palestinians } \\
\text { residing in } \\
\text { Nablus }\end{array}$ & $\begin{array}{l}\text { Saleh et al., } \\
\text { (2018) [36] }\end{array}$ & $\begin{array}{l}N=2001, \text { female } \\
\text { university students }\end{array}$ & $\begin{array}{l}\text { Cross } \\
\text { sectional }\end{array}$ & $\begin{array}{l}\text { EAT } 26 \\
\text { SCOFF }\end{array}$ & $\begin{array}{l}\text { BMI: association between BMI } \\
\text { and EAT score. Age: negative } \\
\text { association between age and } \\
\text { EAT score }\end{array}$ & $\begin{array}{l}\text { BMI: } r=0.173, p<0.011 \\
\text { age: } r=-0.058, p=.008\end{array}$ \\
\hline Qatar & $\begin{array}{l}\text { Bener \& } \\
\text { Kamal (2006) } \\
{[35]}\end{array}$ & $\begin{array}{l}N=566, \text { female, age } 14- \\
19\end{array}$ & $\begin{array}{l}\text { Cross } \\
\text { sectional }\end{array}$ & $\begin{array}{l}\text { Adolescent } \\
\text { dieting scale }\end{array}$ & BMI associated with dieting & $p=0.045$ \\
\hline Qatar & $\begin{array}{l}\text { Bener, Kamal, } \\
\text { Tewfik, \& } \\
\text { Sabuncuoglu } \\
\text { (2006) [35] }\end{array}$ & $N=800$, male, age 14-19 & $\begin{array}{l}\text { Case } \\
\text { control } \\
\text { (dieting) }\end{array}$ & $\begin{array}{l}\text { Adolescent } \\
\text { dieting scale } \\
\text { Self- reporting } \\
\text { questionnaire }\end{array}$ & $\begin{array}{l}\text { Obesity: } 34 \% \text { of dieters was } \\
\text { overweight, TV was diet } \\
\text { source }(61.7 \%)\end{array}$ & $p=0.014$ \\
\hline Country & $\begin{array}{l}\text { Authors } \\
\text { (year) }\end{array}$ & Participants & $\begin{array}{l}\text { Study } \\
\text { design }\end{array}$ & Measures & Risk factors & $M(S D) / O R[95 \% \mathrm{Cl}] / r$ \\
\hline Qatar & $\begin{array}{l}\text { Musaiger, } \\
\text { Shahbeek, \& } \\
\text { Al-Mannai } \\
\text { (2004) [15] }\end{array}$ & $\begin{array}{l}N=535, \text { male, age } 20-67, \\
\text { primary health care } \\
\text { center visitors }\end{array}$ & $\begin{array}{l}\text { Cross } \\
\text { sectional }\end{array}$ & $\begin{array}{l}\text { 9- figure } \\
\text { silhouettes }\end{array}$ & $\begin{array}{l}\text { Age and education } \\
\text { associated with desire to be } \\
\text { thin }\end{array}$ & $\begin{array}{l}\text { Association with desire to be } \\
\text { thin: education: } p=0.0001 \\
\text { age }>40 \text { years } p=0.0001\end{array}$ \\
\hline $\begin{array}{l}\text { Qatar and } \\
\text { Lebanon }\end{array}$ & $\begin{array}{l}\text { Kronfol et al., } \\
\text { (2018) [55] }\end{array}$ & $\begin{array}{l}N=1841,167 \text { Lebanon, } \\
785 \text { Qatar, } 889 \text { USA } \\
\text { university students }\end{array}$ & $\begin{array}{l}\text { Cross } \\
\text { sectional }\end{array}$ & SCOFF & $\begin{array}{l}\text { Risk factors: female gender, } \\
\text { financial difficulties }\end{array}$ & $p<0.001$ \\
\hline Saudi Arabia & $\begin{array}{l}\text { Al- Subaie } \\
(2000)[87]\end{array}$ & $\begin{array}{l}N=1179, \text { female, mean } \\
\text { age }=16.1\end{array}$ & $\begin{array}{l}\text { Cross } \\
\text { sectional }\end{array}$ & EDI 2 DT & $\begin{array}{l}\text { BMI, speaking a Western } \\
\text { language and lived in a } \\
\text { Western country and SES } \\
\text { associated with dieting } \\
\text { behavior and drive for } \\
\text { thinness }\end{array}$ & $\begin{array}{l}\text { BMI: } \times 2(3)=97.59, p=0.0001, \\
\text { western language } \times 2(1)=8.9, \\
p=0.002, \text { lived in western } \\
\text { country } \times 2(1)=10.3, p= \\
0.001, \text { SES } \times 2(4)=12.32, p= \\
0.015\end{array}$ \\
\hline Saudi Arabia & $\begin{array}{l}\text { Fallatah et al., } \\
\text { (2015) [66] }\end{array}$ & $\begin{array}{l}N=425, \text { female, age } 15- \\
18\end{array}$ & $\begin{array}{l}\text { Cross } \\
\text { sectional }\end{array}$ & EAT 26 & $\begin{array}{l}\text { Association between BMI and } \\
\text { disturbed eating behavior }\end{array}$ & $\begin{array}{l}t=3.095, p<0.0001, \mathrm{df} \text { not } \\
\text { reported }\end{array}$ \\
\hline Saudi Arabia & $\begin{array}{l}\text { Khalaf, } \\
\text { Westergren, } \\
\text { Berggren, } \\
\text { Ekblom, \& Al- } \\
\text { Hazzaa (2015) } \\
\text { [95] }\end{array}$ & $\begin{array}{l}N=663, \text { female, mean } \\
\text { age }=20.4 \text {, university } \\
\text { students }\end{array}$ & $\begin{array}{l}\text { Cross } \\
\text { sectional }\end{array}$ & $\begin{array}{l}\text { Self-developed } \\
\text { questionnaire }\end{array}$ & $\begin{array}{l}\text { Weight status: } 19.2 \% \\
\text { underweight, } 56.9 \% \text { normal } \\
\text { weight, } 23.8 \% \text { overweight/ } \\
\text { obesity, BMI was associated } \\
\text { with increased affluence }\end{array}$ & $p=0.032$ \\
\hline Saudi Arabia & $\begin{array}{l}\text { Rasheed } \\
\text { (1998) [68] }\end{array}$ & $\begin{array}{l}N=144, \text { female, } 74 \\
\text { Obese, } 70 \text { non- obese, } \\
\text { age } 15-55\end{array}$ & $\begin{array}{l}\text { Case } \\
\text { control } \\
\text { study } \\
\text { (obesity) }\end{array}$ & $\begin{array}{l}\text { Adapted eating } \\
\text { and exercise } \\
\text { behavior } \\
\text { questionnaire }\end{array}$ & $\begin{array}{l}\text { Increased affluence: higher } \\
\text { affluence leads to slimmer } \\
\text { ideal body ( } 81 \%) \text { and } \\
\text { overestimation of own body } \\
\text { weight ( } 29 \%), \text { illiteracy more } \\
\text { common in obese group } \\
(21.9 \%) \text {. }\end{array}$ & $p<0.05$ \\
\hline UAE & $\begin{array}{l}\text { Eapen et al., } \\
\text { (2006) [20] }\end{array}$ & $\begin{array}{l}N=495, \text { female, age } 13- \\
18\end{array}$ & $\begin{array}{l}\text { Cross } \\
\text { sectional }\end{array}$ & EAT 40 & $\begin{array}{l}\text { BMI, age, Western TV } \\
\text { associated with elevated EAT } \\
\text { scores. }\end{array}$ & $p<0.0001$ \\
\hline UAE & $\begin{array}{l}\text { O'Hara et al., } \\
\text { (2016) [74] }\end{array}$ & $\begin{array}{l}N=420, \text { female, mean } \\
\text { age }=23.12 \text {, university } \\
\text { students }\end{array}$ & $\begin{array}{l}\text { Cross } \\
\text { sectional }\end{array}$ & $\begin{array}{l}\text { EAT } 26 \\
\text { Teasing } \\
\text { frequency from } \\
\text { Project eating } \\
\text { attitudes and } \\
\text { teens } \\
\text { Weight and } \\
\text { body related } \\
\text { shame and guilt }\end{array}$ & $\begin{array}{l}\text { Internalized weight stigma } \\
\text { and teased with weight } \\
\text { associated with elevated EAT } \\
\text { scores }\end{array}$ & $\begin{array}{l}\text { Internalized weight stigma: } \\
r=0.43, p<0.001, \text { teased } \\
\text { with weight: } r=0.19, p= \\
0.008\end{array}$ \\
\hline
\end{tabular}


Table 4 Summary of studies reporting correlates of eating disorders and eating disorder-related variables in the Arab world (Continued)

\begin{tabular}{|c|c|c|c|c|c|c|}
\hline $\begin{array}{l}\text { Country/ } \\
\text { Population }\end{array}$ & $\begin{array}{l}\text { Authors } \\
\text { (year) }\end{array}$ & Participants & $\begin{array}{l}\text { Study } \\
\text { design }\end{array}$ & Measures & Risk factors & $M(S D) /$ OR $[95 \% \mathrm{Cl}] / r$ \\
\hline & & & & scale & & \\
\hline UAE & $\begin{array}{l}\text { Schulte \& } \\
\text { Thomas } \\
\text { (2013) [89] }\end{array}$ & $\begin{array}{l}N=361,77 \text { male, } 284 \\
\text { female, age } 11-19, \\
\text { university students }\end{array}$ & $\begin{array}{l}\text { Cross } \\
\text { sectional }\end{array}$ & EAT 26 & $\begin{array}{l}\text { Weight status: overweight: } \\
18.6 \% \text {, obesity } 9.2 \% \\
\text { BMI associated with EAT } \\
\text { scores in females }\end{array}$ & BMI: $r=0.184, p=0.005$ \\
\hline UAE & $\begin{array}{l}\text { Schulte } \\
\text { (2016) [90] }\end{array}$ & $\begin{array}{l}N=236, \text { mean age }= \\
19.78\end{array}$ & $\begin{array}{l}\text { Cross } \\
\text { sectional }\end{array}$ & $\begin{array}{l}\text { Body esteem } \\
\text { scale } \\
\text { PSS } \\
\text { Emotional } \\
\text { eating scale, } \\
\text { Weight and } \\
\text { body related } \\
\text { shame and guilt } \\
\text { scale }\end{array}$ & $\begin{array}{l}\text { After correcting for BMI } \\
\text { association between } \\
\text { perceived stress and binge } \\
\text { eating }\end{array}$ & $p=0.043$ \\
\hline UAE & $\begin{array}{l}\text { Thomas, } \\
\text { Quadflieg, \& } \\
\text { O'Hara (2016) } \\
{[40]}\end{array}$ & $\begin{array}{l}N=94, \text { female, university } \\
\text { students }\end{array}$ & $\begin{array}{l}\text { Cross } \\
\text { sectional }\end{array}$ & EAT 26 & $\begin{array}{l}\text { Implicit out group preference } \\
\text { associated with elevated EAT } \\
\text { scores }\end{array}$ & $t[91]=2.83, p<0.001$ \\
\hline UAE & $\begin{array}{l}\text { Thomas, } \\
\text { O'Hara, et al., } \\
\text { (2018) [53] }\end{array}$ & $\begin{array}{l}N=1069, \text { female, } \\
\text { university students }\end{array}$ & $\begin{array}{l}\text { Cross } \\
\text { sectional }\end{array}$ & EAT 26 & $\begin{array}{l}\text { Religiosity: small effect size } \\
\text { for religiosity in the group } \\
\text { that scored above clinical cut } \\
\text { off on the EAT }\end{array}$ & $\begin{array}{l}U=91,660, p<0.001, r=- \\
0.12\end{array}$ \\
\hline UAE & $\begin{array}{l}\text { Thomas, } \\
\text { O'Hara, et al., } \\
\text { (2018) [18] }\end{array}$ & $\begin{array}{l}N=209, \text { female, } \\
\text { university students }\end{array}$ & $\begin{array}{l}\text { Cross } \\
\text { sectional }\end{array}$ & EAT 26 & $\begin{array}{l}\text { Westernization: small effect } \\
\text { size for out group positivity } \\
\text { and higher Western } \\
\text { acculturation in the group } \\
\text { that scored above clinical cut } \\
\text { off on the EAT }\end{array}$ & $\begin{array}{l}\text { Out group positivity: } t(206)= \\
2.49, p=0.013, d=0.36 \\
\text { Western acculturation: } \\
t(206)=3.13, p=0.002, d= \\
0.46\end{array}$ \\
\hline
\end{tabular}

Note: BSQ Body Shape Questionnaire, EAT Eating Attitude Test, EDE-Q Eating Disorder Examination Questionnaire, FRS Figure Rating Scale, EDI 2 DT Eating Disorders Inventory 2 Drive for Thinness Scale, SCID Structured Clinical Interview for DSM IV, SCOFF Sick, Control, One, Stone, Fat, Food

oil boom in the 1970s and the consequent increased affluence have contributed to elevated levels of noncommunicable diseases including the development of ED-related variables, ED symptoms and EDs [18, 3840]. In the included studies sociocultural changes were associated with changes in types of food available (higher in salt, fat and sugar) and therefore increased prevalence of obesity [34, 100-102]. Obesity was associated with dieting behavior, compensatory behavior, body dissatisfaction and binge eating behavior [13, 20, 23, 26, 70, 76, 85, 103]. Another implication of the sociocultural changes was the shift from admiration of a curvy body [23] to that of a thin body [10, 30, 80, 94], and the desire to be thin was associated with compensatory behavior, binge eating behavior, dieting behavior, and body dissatisfaction $[13,20,23,26,70,76,85,103]$. The thin ideal was especially prevalent among adults $[10,13-15,20$, 87, 89], higher educated, increased affluence Arabs [38, 104], and Arabs who reported Western influences [18, 30]. Interestingly, societies dealing with faster industrialization appeared more vulnerable to Western influences $[18,40]$ and displayed greater risk for EDs [12, 29, 76, 77]. However, Qatari females appeared minimally affected by the thin ideal [15]. There were indications that culture was associated with symptomatology $[29,52,58,64]$, and the somatic symptom presentation $[52,58]$ was in line with other countries dealing with rapid sociocultural changes $[59,105,106]$.

In conclusion, female Arabs were most at risk for EDs and ED symptoms $[20,26,29,52,53,58,67]$. The high rates of obesity $[34,100-102]$ and the desire to be thin were associated with other ED-related variables, ED symptoms and high risk for EDs [13, 20, 23, 26, 70, 76, $85,103]$. Increased affluence and media use were correlates of the desire to be thin.

\section{Clinical impact}

Development of this synthesis of EDs in the Arab world has important clinical implications, as most studies examining ED prevalence used Western assessment tools [90]. Such utilization of potentially insensitive assessment tools and potentially inappropriate norms may lead to underestimation of symptom severity [107], elevated rates of undiagnosed EDs, and to a lack of knowledge and (public) awareness about EDs and its correlates [108]. This in turn hampers timely and proper treatment of EDs [69]. In the Arab world, the situation is further 
complicated by the lack of specialized therapists and treatment facilities [97].

Another problematic aspect of the lack of popular knowledge about EDs is stigmatization, leading to delayed help seeking [108]. Since it is culturally unacceptable to discuss personal matters with non-family members, only $0.3 \%$ of Arabs with an ED seek help, compared to $20 \%$ of Westerners who do so [70]. More knowledge about EDs might decrease the current preference for self- or family treatment, and therefore stigma associated with psychotherapy [70]. In order to increase knowledge psycho-education can be offered at highschools, including a parental program. Once college and high-school social workers are better educated in recognizing EDs they can refer their students to mental health care clinics. In addition, overweight Arabs tend to seek bariatric surgery, while currently they are in general not screened for EDs. Screening for EDs and refer Arabs at high risk for EDs might reduce delayed help seeking. Last, to decrease stigmatization, it might be beneficial to let influencers share their personal story of recovery of an ED, since Arabs are extensive social media users.

Two studies in Oman reported that, as in several Asian studies [59, 105, 106], EDs were explained somatically rather than psychiatrically $[52,58]$. This may lead to a failure to accurately recognize behaviors as ED symptoms, and so to delayed help-seeking and greater symptom severity $[109,110]$. In addition, in Saudi Arabia people with EDs tended to seek help only after experiencing somatic complaints, such as kidney failure or diabetes mellitus.

Although EDs have been studied in the Arab world, there are methodological shortcomings to many of the studies currently available. Arabs who display an increased risk for EDs, are likely to benefit from preventative programs, as selective prevention programs display larger effects than universal prevention programs [49, 111-113]. The risk groups in the Arab world are individuals with elevated levels of body dissatisfaction, desire to be thin, and self-reported dieting $[13,20,23,26,70,76$, $85,103]$. In addition, the more high affluent societies and Arabs with extensive (social) media use, and more prone to Western influences are also at risk [38, 104]. Counteracting the elevated prevalence of obesity in order to prevent associated health risks [80], accompanied by psycho-education in order to prevent maladaptive weight-loss strategies, may be beneficial.

\section{Future directions}

Most assessment tools included in this synthesis have been devised for use in Western populations. Due to the potential bias, it is recommended to use ED-screening instruments adapted for use in the Arab world. Future research might therefore consider the development and validation of cultural sensitive assessment tools [66]. One relevant adaptation of self- report measures is that fasting may be motivated by religion $[77,96]$ and should only be considered pathological if motivated by weight and shape concern. Other relevant adaptations in some Arab countries might relate to the strict gender separations and related cultural norms and behaviors, e.g., a proper attention to distinguishing between situations where females do and don't cover themselves [77].

Another potential bias of the studies included in this synthesis is the usage of Western norms. In general, scores on ED-screening instruments are significantly lower in Western populations than in Arab populations [28, 80, 114, 115], usage of Western norms might therefore reflect bias. While some assessment tools have been validated in other Arab countries, they potentially may not be sensitive to nuances across various Arab communities. Efforts to assess EDs in the Arab world have therefore potentially been limited due to the absence of psychometric validation of assessment tools, especially in clinical samples. In order to understand the culture, validation studies should be supplemented with interview data. A culturally sensitive assessment tool will help minimize inconsistencies found among studies in the Arab world.

To elucidate correlates and ED-related variables, researchers need to examine levels of acculturation, internalization of the thin ideal, increased affluence and media exposure, as they may mediate body image dissatisfaction and disturbed eating behavior. As most studies included in this synthesis were conducted in countries dealing with rapid sociocultural changes, additional studies in the Arab world with limited exposure to Western influences and limited industrialization are necessary $[116,117]$.

Recently, studies are beginning to address the possible somatic symptom presentation of EDs in the Arab world $[52,58]$. We recommend that, during diagnostic interviews, clinicians pay attention to the possibility of this understudied phenomena. In addition, it is worth further investigating the somatic attribution of EDs in the Arab world. Confirmation of this theory will facilitate recognition of EDs in the Arab world.

\section{Supplementary information}

Supplementary information accompanies this paper at https://doi.org/10. 1186/s40337-020-00336-X.

\section{Additional file 1.}

\section{Abbreviations}

AN: Anorexia Nervosa; BED: Binge Eating Disorder; BES: Binge Eating Scale; BMI: Body Mass Index; BN: Bulimia Nervosa; BSQ: Body Shape Questionnaire; EAT: Eating Attitudes Test; ED: Eating Disorders; EDE-Q: Eating Disorder Examination Questionnaire; EDI 2 DT: Eating Disorders Inventory 2 Drive for Thinness Scale; FRS: Figure Rating Scale; GAD-7: General Anxiety Disorder questionnaire; OCI-R: Obsessive Compulsive Inventory; OSFED: Other 
Specified Feeding or Eating Disorder; PRISMA: Preferred Reporting Items for Systematic reviews and Meta-Analyses; PSS: Perceived Stress Scale; SCID: Structured Clinical Interview for DSM IV; SCOFF: Sick, Control, One, Stone, Fat, Food; UAE: United Arab Emirates

\section{Acknowledgements}

We gratefully acknowledge the help of Ian Charles Whyte as an English corrector.

\section{Authors' contributions}

All authors contributed to the above manuscript and gave their approval. $\mathrm{BM}$ was responsible for examining literature and writing the manuscript. EdB and EvF contributed to writing the manuscript.

\section{Funding}

Not applicable.

\section{Availability of data and materials}

Not applicable.

\section{Ethics approval and consent to participate}

Ethical approval and informed consent for this type of study is not required.

\section{Consent for publication}

Not applicable.

\section{Competing interests}

All authors declare that there are no competing interests.

\section{Author details}

${ }^{1}$ Novarum Center for Eating Disorders \& Obesity, Jacob Obrechtstraat 92, 1071, KR, Amsterdam, the Netherlands. ${ }^{2}$ Rivierduinen Eating Disorders Ursula, Sandifortdreef 19, 2333 ZZ Leiden, Netherlands. ${ }^{3}$ Department of Psychiatry, Leiden University Medical Center, Albinusdreef 2, 2333 ZA Leiden, Netherlands. ${ }^{4}$ Arkin Mental Health Institute, research department, Klaprozenweg 111, 1033 NN Amsterdam, the Netherlands. ${ }^{5}$ Leiden University, Section Clinical Psychology, Wassenaarseweg 52, 2333 AK Leiden, the Netherlands.

Received: 28 March 2020 Accepted: 7 October 2020

Published online: 06 November 2020

\section{References}

1. Hay P, Girosi F, Mond J. Prevalence and sociodemographic correlates of DSM-5 eating disorders in the Australian population. J Eat Disord. 2015;3(1): 19

2. Brody ML, Masheb RM, Grilo CM. Treatment preferences of patients with binge eating disorder. Int J Eat Disord. 2005:37(4):352-6.

3. Watson HJ, Allen K, Fursland A, Byrne SM, Nathan PR. Does enhanced cognitive behaviour therapy for eating disorders improve quality of life? Eur Eat Disord Rev. 2012;20(5):393-9.

4. Wilson GT, Becker CB, Heffernan K. Eating disorders. Child psychopathology, 2nd ed. New York, NY, US: Guilford Press; 2003. p. 687-715.

5. Smink FR, van Hoeken D, Hoek HW. Epidemiology of eating disorders: incidence, prevalence and mortality rates. Curr Psychiatry Rep. 2012;14(4): 406-14

6. Gordon RA. Eating disorders east and west: a culture-bound syndrome unbound. Int J Eat Disord. 2001;1:16.

7. Pike KM, Hoek HW, Dunne PE. Cultural trends and eating disorders. Curr Opin Psychiatry. 2014;27(6):436-42.

8. Pike K, Dunne P. The rise of eating disorders in Asia: a review. J Eat Disord. 2015:3(1):1-14.

9. Lewis-Fernández R, Kleinman A. Culture, personality, and psychopathology. J Abnorm Psychol. 1994;103(1):67-71.

10. Ford KA, Dolan BM, Evans C. Cultural factors in the eating disorders: a study of body shape preferences of Arab students. J Psychosom Res. 1990;34(5): 501-7.

11. Dolan B, Ford K. Binge eating and dietary restraint: a cross-cultural analysis. Int J Eat Disord. 1991;10(3):345-53.
12. Nasser M. Comparative study of the prevalence of abnormal eating attitudes among Arab female students of both London and Cairo universities. Psychol Med. 1986;16(3):621-5.

13. Al Subaie AS. Eating attitude test in Arabic. Psychometric features and normative data. Ann Saudi Med. 1999;19:447-53.

14. Thomas J, Khan S, Abdulrahman AA. Eating attitudes and body image concerns among female university students in the United Arab Emirates. Appetite. 2010;54(3):595-8.

15. Musaiger AO, Shahbeek NE, Al-Mannai M. The role of social factors and weight status in ideal body shape preferences as perceived by Arab women. J Biosoc Sci. 2004;36(6):699-707.

16. Thomas JJ, Lee S, Becker AE. Updates in the epidemiology of eating disorders in Asia and the Pacific. Curr Opin Psychiatry. 2016:29(6):354-62.

17. Keel PK, Klump KL. Are eating disorders culture- bound syndromes? Implications for conceptualizing their etiology. Psychol Bull. 2003;129(5):747-69.

18. Thomas, J., O'Hara, L., Quadflieg, S., \& Weissgerber, S. C. (2018). Acculturation, out-group positivity and eating disorders symptoms among Emirati women. Eat Weight Disord, 23(2), 241-246. https://doi.org/10.1007/s40519-016-0358-5.

19. van Son GE, van Hoeken D, Bartelds Al, van Furth EF, Hoek HW. Urbanisation and the incidence of eating disorders. Br J Psychiatry. 2006; 189:562-3.

20. Eapen VM, Mabrouk AA, Bin Othman S. Disordered eating attitudes and symptomatology among adolescent girls in the United Arab Emirates. Eat Behav. 2006;7(1):53-60

21. WHO. Country cooperation strategy for WHO and the United Arab Emirates 2005-2009. from World Health Organisation; 2006.

22. Fox JW, Mourtada-Sabbah N. The Arab Gulf region: Traditionalism globalized or globalization traditionalized? Globalization and the Gulf. London: Routledge; 2006. p. 13-70

23. Zeeni N, Gharibeh N, Katsounari I. The influence of sociocultural factors on the eating attitudes of Lebanese and Cypriot students: a cross-cultural study. J Hum Nutr Diet. 2013;26(Suppl 1):45-52.

24. Salant T, Lauderdale DS. Measuring culture: a critical review of acculturation and health in Asian immigrant populations. Soc Sci Med. 2003:57(1):71-90.

25. Ahmed R. Prophetic traditions and modern medicine in the Middle East: resurrection, reinterpretation, and reconstruction. J Am Oriental Soc. 2012 132(4):657-73.

26. Musaiger AO, Al-Mannai M, Tayyem R, Al-Lalla O, Ali EY, Kalam F, et al. Risk of disordered eating attitudes among adolescents in seven Arab countries by gender and obesity: a cross-cultural study. Appetite. 2013:60(1):162-7.

27. Musaiger AO, Al-Mannai M. Association between exposure to media and body weight concern among female university students in five Arab countries: a preliminary cross- cultural study. J Biosoc Sci. 2014;46(2):240-7.

28. Eladawi N, Helal R, Niazy NA, Abdelsalam S. Prevalence and associated factors of eating disorders in weight management centers in Tanta, Egypt Chin Med J. 2018;131(1):50-5.

29. Al Adawi S, Dorvlo AS, Burke DT, Al Bahlani S, Martin RG, Al-Ismaily S. Presence and severity of anorexia and bulimia among male and female Omani and non Omani adolescents. J Am Acad Child Adolescent Psychiatry. 2002;4(9):1124-30

30. Thomas J, Al Marzooqi FH, Tahboub-Schulte S, Furber SW. Changing physical appearance preferences in the United Arab Emirates. Mental Health Religion Cult. 2014:17(6):594-600.

31. Lester R. Commentary: eating disorders and the problem of "culture" in acculturation. Cult Med Psychiatry. 2004:28(4):607-15.

32. Pavlova $B$, Uher $R$, Papezova H. It would not have happened to me at home: qualitative exploration of sujourns abroad and eating disorders in young Czech women. Eur Eat Disord Rev. 2008;16:207-14.

33. Mourtada-Sabbah N, Al-Mutawa M, Fox JW, Walters T. Media as social matrix in the United Arab Emirates. In: Popular culture and political identity in the Arab Gulf States; 2008. p. 121-42

34. Swain P. Anorexia nervosa and bulimia nervosa: new research. Hauppauge, NY: Nova Publishers; 2006

35. Bener A, Kamal A, Tewfik I, Sabuncuoglu O. Prevalence of dieting overweight, body image satisfaction and associated psychological problems in adolescent boys. J Nutr Food Sci. 2006;36(5):295-304.

36. Saleh RN, Salameh RA, Yhya HH, Sweileh WM. Disordered eating attitudes in female students of An-Najah National University: a cross-sectional study. J Eat Disord. 2018;6:16

37. Ghubash R, Daradkeh TK, Al-Muzafari SMA, El-Manssori ME, Abou-Saleh MT. Al-Ain community psychiatric survey IV: socio-cultural changes 
(traditionality-liberalism) and prevalence of psychiatric disorders. Soc Psychiatry Psychiatr Epidemiol. 2001;36(11):565-70.

38. Pavlova B, Uher R, Dragomirecka E, Papezova H. Trends in hospital admissions for eating disorders in a country undergoing a socio-cultural transition, the Czech Republic 1981-2005. Soc Psychiatry Psychiatr Epidemiol. 2010;45(5):541-50.

39. Nasser M. Screening for abnormal eating attitudes in a population of Egyptian secondary school girls. Soc Psychiatry Psychiatr Epidemiol. 1994; 29(1):25-30.

40. Thomas J, Quadflieg S, O'Hara L. Implicit out-group preference is associated with eating disorders symptoms amongst Emirati females. Eat Behav. 2016; 21:48-53.

41. Kraemer HC, Kazdin AE, Offord DR, Kessler RC, Jensen PS, Kupfer DJ. Coming to terms with the terms of risk. Arch Gen Psychiatry. 1997;54(4):337-43.

42. Jacobi C, Hayward C, de Zwaan M, Kraemer HC, Agras WS. Coming to terms with risk factors for eating disorders: application of risk terminology and suggestions for a general taxonomy. Psychol Bull. 2004;130(1):19-65.

43. Stice $\mathrm{E}, \mathrm{Ng}$ J, Shaw H. Risk factors and prodromal eating pathology. J Child Psychol Psychiatry. 2010;51(4):518-25.

44. Jacobi C, Abascal L, Taylor CB. Screening for eating disorders and high-risk behavior: caution. Int J Eat Disord. 2004;36(3):280-95.

45. Stice E, Marti CN, Spoor S, Presnell K, Shaw H. Dissonance and healthy weight eating disorder prevention programs: long-term effects from a randomized efficacy trial. J Consult Clin Psychol. 2008;76(2):329-40.

46. Nakai Y, Nin K, Noma S, Teramukai S, Fujikawa K, Wonderlich SA. Changing profile of eating disorders between 1963 and 2004 in a Japanese sample. Int J Eat Disord. 2018;51(8):953-8.

47. Association AP. Diagnostic and statistical manual of mental disorders fifth edition: DSM- 5. Washington DC: American Psychiatric Publishing; 2013.

48. Kraemer HC, Stice E, Kazdin A, Offord D, Kupfer D. How do risk factors work together? Mediators, moderators, and independent, overlapping, and proxy risk factors. Am J Psychiatr. 2001;158(6):848-56.

49. Stice $E$, Shaw $H$, Marti CN. A meta-analytic review of eating disorder prevention programs: encouraging findings. Annu Rev Clin Psychol. 2007;3: 207-31.

50. Hoek HW. Review of the worldwide epidemiology of eating disorders. Curr Opin Psychiatry. 2016;29(6):336-9.

51. Smink FR, van Hoeken D, Hoek HW. Epidemiology, course, and outcome of eating disorders. Curr Opin Psychiatry. 2013;26(6):543-8.

52. Viernes N, Zaidan ZA, Dorvlo AS, Kayano M, Yoishiuchi K, Kumano H, et al. Tendency toward deliberate food restriction, fear of fatness and somatic attribution in cross-cultural samples. Eat Behav. 2007;8(3):407-17.

53. Thomas J, O'Hara L, Tahboub-Schulte S, Grey I, Chowdhury N. Holy anorexia: eating disorders symptomatology and religiosity among Muslim women in the United Arab Emirates. Psychiatry Res. 2018;260:495-9.

54. Aoun A, Azzam J, Jabbour FE, Hlais S, Daham D, Amm CE, et al. Validation of the Arabic version of the SCOFF questionnaire for the screening of eating disorders. East Mediterr Health J. 2015;21(5):326-31.

55. Kronfol Z, Khalifa B, Khoury B, Omar O, Daouk S, deWitt JP, et al. Selected psychiatric problems among college students in two Arab countries: comparison with the USA. BMC Psychiatry. 2018;18(1):1-9 Available from: https://bmcpsychiatry.biomedcentral.com/track/pdf/10.11 86/s12888-018-1718-7.

56. van Hoeken D, Burns JK, Hoek HW. Epidemiology of eating disorders in Africa. Curr Opin Psychiatry. 2016;29(6):372-7.

57. Hoek HW, van Hoeken D. Review of the prevalence and incidence of eating disorders. Int J Eat Disord. 2003;34(4):383-96.

58. Kayano M, Yoshiuchi K, Al-Adawi S, Viernes N, Dorvlo AS, Kumano H, et al. Eating attitudes and body dissatisfaction in adolescents: cross-cultural study. Psychiatry Clin Neurosci. 2008;62(1):17-25.

59. Lee $S$, Lee AM, Ngai E, Lee DTS, Wing YK. Rationales for food refusal in Chinese patients with anorexia nervosa. Int J Eat Disord. 2001;29(2):224-9.

60. Smink FR, van Hoeken D, Donker GA, Susser ES, Oldehinkel AJ, Hoek HW. Three decades of eating disorders in Dutch primary care: decreasing incidence of bulimia nervosa but not of anorexia nervosa. Psychol Med. 2016;46(6):1189-96.

61. Ricciardelli LA, McCabe MP, Williams RJ, Thompson JK. The role of ethnicity and culture in body image and disordered eating among males. Clin Psychol Rev. 2007;27(5):582-606.

62. WHO. WHO's Eastern Mediterranean Region 2020 [Available from: http:// www.emro.who.int/countries.html. Accessed May 62020.
63. infoplease.com. Middle East Map 2020 [The Middle East is a loosely defined geographic region; the countries listed are generally considered part of the Middle East. These Middle East countries are part of the Asian continent, with the exception of Egypt, which is part of Africa, and the northwestern part of Turkey, which is part of the European landmass.]. Available from: https://www.infoplease.com/atlas/middle-east. Accessed May 62020.

64. Abdollahi P, Mann T. Eating disorder symptoms and body image concerns in Iran: comparisons between Iranian women in Iran and in America. Int J Eat Disord. 2001;30(3):259-68.

65. Berg KC, Stiles-Shields EC, Swanson SA, Peterson CB, Lebow J, Le Grange D. Diagnostic concordance of the interview and questionnaire versions of the eating disorder examination. Int J Eat Disord. 2012;45(7):850-5.

66. Fallatah A, Al-Hemairy M, Al-Ghamidi H. Eating disorders among female adolescents in Jeddah. C COOP. 2015;138. Retrieved from https://core.ac.uk/ download/pdf/35341517.pdf\#page $=148$.

67. Bano R, Al Shammari E, Banu S. A study on the prevalence and severity of eating disorders among the young population of hail city in Saudi Arabia. Medical Science. 2013;2(6):159-73.

68. Rasheed P. Perception of body weight and self-reported eating and exercise behaviour among obese and non-obese women in Saudi Arabia. Public Health. 1998;112(6):409-14. https://doi.org/10.1038/sj.ph.1900479.

69. Alkhadari S, Alsabbrri AO, Mohammad IHA, Atwan AA, Alqudaihi F, Zahid MA. Prevalence of psychiatric morbidity in the primary health clinic attendees in Kuwait. J Affect Disord. 2016;195:15-20.

70. Latzer Y, Azaiza F, Tzischinsky O. Eating attitudes and dieting behavior among religious subgroups of Israeli- Arab adolescent females. J Relig Health. 2009;48(2):189-99.

71. Modesti PA, Reboldi G, Cappuccio FP, Agyemang C, Remuzzi G, Rapi S, et al. Panethnic differences in blood pressure in Europe: a systematic review and meta-analysis. PLoS One. 2016;11(1):e0147601.

72. Moher D, Tetzlaff J, Liberati A, Altman DG. Preferred reporting items for systematic reviews and meta-analyses: the PRISMA statement. Int I Surg. 2010;8(5):336-41.

73. Musaiger AO. Overweight and obesity in eastern mediterranean region: prevalence and possible causes. J Obes. 2011;2011:407237.

74. O'Hara L, Tahoub-Schulte S, Thomas J. Weight- related teasing and internalized weight stigma predict abnormal eating attitudes and behaviours in Emirati female university students. Appetite. 2016;102:44-50.

75. Musaiger AO, Al-Mannai M. Role of obesity and media in body weight concern among female university students in Kuwait. Eat Behav. 2013;14(1): 229-32.

76. Raouf M, Ebrahimi H, Jafarabadi MA, Malek A, Kheirrodin JB. Prevalence of eating disorders among adolescents in the northwest of Iran. Iran Red Crescent Med J. 2015;17(10):1-5.

77. Mousa TM, Al-Domi HA, Mashal RH, Jibril MAK. Eating disturbances among adolescent schoolgirls in Jordan. Appetite. 2010;54(1):196-201.

78. al-Subaie A, al-Shammari S, Bamgboye E, al-Sabhan K, al-Shehri S, Bannah AR. Validity of the Arabic version of the eating attitude test. Int J Eat Disord. 1996;20(3):321-4.

79. Musaiger AO, Al-Mannai M, Al-Lalla O. Risk of disordered eating attitudes among male adolescents in five emirates of the United Arab Emirates. Int J Eat Disord. 2014;47(8):898-900.

80. Madanat HN, Brown RB, Hawks SR. The impact of body mass index and Western advertising and media on eating style, body image and nutrition transition among Jordanian women. Public Health Nutr. 2007; 10(10):1039-46.

81. al-Subaie AS, Bamgboye E, al-Shammari S, al-Sabhan KN, al-Shehri SN, Bannah AR. Validity of the Arabic version of the eating disorders inventory (EDI). Br J Psychiatry. 1996;168(5):636-40.

82. Al-Sendi A, Shetty P, Musaiger A. Body weight perception among Bahraini adolescents. J Child Care Health Dev. 2004;30(4):369-76.

83. Madanat H, Hawks SR, Angeles HN. Obesity and boy size preferences of Jordanian women. Health Educ Behav. 2011;38(1):91-8.

84. Mousa TY, Mashal RH, Al-Domi HA, Jibril MA. Body image dissatisfaction among adolescent schoolgirls in Jordan. Body Image. 2010;7(1):46-50.

85. Zawawi JA. Relationships between body mass index, body image dissatisfaction and psychological distress among fitness center female users in Zarqa-Jordan. Int J Human Soc Sci. 2014;4(11):1.

86. Mariam E, Dalal A, Tasleem AZ, Stan K. Disordered eating attitudes correlate with body dissatisfaction among Kuwaiti male college students. Journal of Eating disorders. 2019;7(1):1-13. https://doi.org/10.1186/s40337-019-0265-z. 
87. Al-Subaie AS. Some Correlates of Dieting Behavior in Saudi Schoolgirls. Int J Eat Disord. 2000;28:242-6.

88. Sawadi H, Bener A, Al Darmaki F. Dieting among Arab girls 2000.

89. Schulte SJ, Thomas J. Relationship between eating pathology, body dissatisfaction and depressive symptoms among male and female adolescents in the United Arab Emirates. Eat Behav. 2013;14(2):157-60.

90. Schulte SJ. Predictors of binge eating in male and female youths in the United Arab Emirates. Appetite. 2016:105:312-9.

91. Mariam, E., Dalal, A., Tasleem, A. Z., \& Stan, K. (2019). Disordered eating attitudes correlate with body dissatisfaction among Kuwaiti male college students. Journal of Eating disorders, 7(1), 1-13. https://doi.org/10.1186/ s40337-019-0265-Z.

92. El-Ghazali S, Ibrahim JM, Kanari B, Ismail NA. The relationship between lifestyle and body mass index among university students in Kuwait. Egypt J Commun Med. 2010;28(1). Retrieved from https://www.scopus.com/record/ display.uri?eid=2-s2.0-84875068472\&origin=inward\&txGid=3da9c121038503 0a9b17e81edf9ded53.

93. Yahia N, El-Ghazale H, Achkar A, Rizk S. Dieting practices and body image perception among Lebanese university students. Asia Pac J Clin Nutr. 2011; 20:21-8.

94. Afifi-Soweid RM, Najem Kteily M, Shedia-Rizkallah M. Preoccupation with weight and disordered eating behaviors of entering students at a university in Lebanon. Int J Eat Disord. 2001;32:52-7.

95. Khalaf, A., Westergren, A., Berggren, V., Ekblom, O., \& Al-Hazzaa, H. M. (2015). Prevalence and association of female weight status and dietary habits with sociodemographic factors: a cross-sectional study in Saudi Arabia. Public Health Nutr, 18(5), 784-796. https://doi.org/10.1017/\$1368980014001797.

96. GCC-Stat (Producer). (2016, 9-11-2016). Health. Retrieved from http://gccstat. org/en/statistics/demographic-and-social/health.

97. Qadan L. Anorexia nervosa: beyond boundaries. Int J Eat Disord. 2009;42(5): 479-81.

98. Mirkim B. Population levels, trends and policies in the Arab region. Challenges and opportunities. Arab Human Development report. USA: United Nations Development Programme; 2012. 17-09-2016.

99. Gerbasi ME, Richards LK, Thomas JJ, Agnew-Blais JC, Thompson-Brenner H, Gilman SE, et al. Globalization and eating disorder risk: peer influence, perceived social norms, and adolescent disordered eating in Fiji. Int J Eat Disord. 2014;47(7):727-37.

100. Goldschmidt AB, Aspen VP, Sinton MM, Tanofsky-Kraff M, Wilfley DE. Disordered eating attitudes and behaviors in overweight youth. Obesity (Silver Spring). 2008;16(2):257-64.

101. Wong Y, Chang YJ, Tsai MR, Liu TW, Lin W. The body image, weight satisfaction, and eating disorder tendency of school children: the 2-year follow-up study. J Am Coll Nutr. 2011;30(2):126-33.

102. Hae-Jeung L, Sanghshin P, Cho-il K, Doo-won C, Jung Sun L, Sun Min O, et al. The association between disturbed eating behavior and socioeconomic status: the Korean adolescent panel survey (OnKPAS). Public Library of science one. 2013:8(3):1-7.

103. Wardle J. Eating style: a validation study of the Dutch eating behaviour questionnaire in normal subjects and women with eating disorders. J Psychosom Res. 1987;31(2):161-9.

104. Abou-Saleh MT, Younis Y, Karim L. Anorexia nervosa in an Arab culture. Int J Eat Disord. 1996;23(2):207-12.

105. Srinivasan TN, Suresh TR, Jayaram V, Fernandez MP. Eating disorders in India. Indian J Psychiatry. 1995;37(1):26-30.

106. Khandelwal SK, Sharan P, Saxena S. Eating disorders: an Indian perspective. Int J Soc Psychiatry. 1995;41(2):132-46.

107. Darcy AM, Doyle AC, Lock J, Peebles R, Doyle P, Le Grange D. The eating disorders examination in adolescent males with anorexia nervosa: how does it compare to adolescent females? Int J Eat Disord. 2012;45(1):110-4.

108. Griffiths S, Mond JM, Murray SB, Thornton C, Touyz S. Stigma resistance in eating disorders. Soc Psychiatry Psychiatr Epidemiol. 2015;50(2):279-87.

109. Mond JM. Eating disorders "mental health literacy": an introduction. J Ment Health. 2014;23(2):51-4

110. Räisänen U, Hunt K. The role of gendered constructions of eating disorders in delayed help-seeking in men: a qualitative interview study. BMJ Open. 2014:4(4):e004342.

111. Bar RJ, Cassin SE, Dionne MM. The long-term impact of an eating disorder prevention program for professional ballet school students: a 15-year follow-up study. Eat Disord. 2017;25(5):375-87.
112. Kindermann S, Moessner M, Ozer F, Bauer S. Associations between eating disorder related symptoms and participants' utilization of an individualized internet-based prevention and early intervention program. Int J Eat Disord. 2017;50(10):1215-21.

113. Stice $E$, Johnson S, Turgon R. Eating disorder prevention. Psychiatric Clin North Am. 2019:42(2):309-18.

114. Melisse B, van Furth $E$, de Beurs $E$. The eating disorder examinationquestionnaire: norms and validity for Saudi nationals; 2020.

115. Fairburn CG, Beglin SJ. Eating Disorder Examination- Questionnaire (6.0); 2008.

116. Campbell B, Pope H, Filiault S. Body image among Ariaal men from northern Kenya. J Cross-Cult Psychol. 2005;36(3):371-9.

117. Yang CF, Gray P, Pope HG Jr. Male body image in Taiwan versus the west: Yanggang Zhiqi meets the Adonis complex. Am J Psychiatry. 2005;162(2): $263-9$

\section{Publisher's Note}

Springer Nature remains neutral with regard to jurisdictional claims in published maps and institutional affiliations.

Ready to submit your research? Choose BMC and benefit from:

- fast, convenient online submission

- thorough peer review by experienced researchers in your field

- rapid publication on acceptance

- support for research data, including large and complex data types

- gold Open Access which fosters wider collaboration and increased citations

- maximum visibility for your research: over $100 \mathrm{M}$ website views per year

At $\mathrm{BMC}$, research is always in progress.

Learn more biomedcentral.com/submissions 